\title{
An Approach to BMBJ-Neutrosophic Hyper-BCK-Ideals of Hyper-BCK-Algebras
}

\author{
Abdelaziz Alsubie (D), ${ }^{1}$ Anas Al-Masarwah $\left(\mathbb{D},{ }^{2}\right.$ Young Bae Jun, ${ }^{3}$ and Abd Ghafur Ahmad ${ }^{2}$ \\ ${ }^{1}$ Department of Basic Sciences, College of Science and Theoretical Studies, Saudi Electronic University, Riyadh, Saudi Arabia \\ ${ }^{2}$ Department of Mathematical Sciences, Faculty of Science and Technology, Universiti Kebangsaan Malaysia, UKM Bangi 43600, \\ Selangor DE, Malaysia \\ ${ }^{3}$ Department of Mathematics Education, Gyeongsang National University, Jinju 52828, Republic of Korea
}

Correspondence should be addressed to Abdelaziz Alsubie; a.alsubie@seu.edu.sa

Received 18 January 2021; Revised 10 April 2021; Accepted 30 April 2021; Published 25 May 2021

Academic Editor: Feng Feng

Copyright ( $\odot 2021$ Abdelaziz Alsubie et al. This is an open access article distributed under the Creative Commons Attribution License, which permits unrestricted use, distribution, and reproduction in any medium, provided the original work is properly cited.

\begin{abstract}
In this article, a new idea of $\mathrm{BMBJ}$-neutrosophic hyper-BCK-algebras is introduced and some of its properties are investigated. Here, BMBJ-neutrosophic hyper-BCK-ideal, BMBJ-neutrosophic weak hyper-BCK-ideal, BMBJ-neutrosophic s-weak hyper$\mathrm{BCK}$-ideal, and BMBJ-neutrosophic strong hyper-BCK-ideal are presented, and some relevant results and relations are indicated. Characterizations of BMBJ-neutrosophic (weak, s-weak, strong) hyper-BCK-ideal are considered. Conditions for a BMBJneutrosophic weak hyper-BCK-ideal to be a BMBJ-neutrosophic s-weak hyper-BCK-ideal are provided. Conditions for an MBJneutrosophic set to be a BMBJ-neutrosophic strong hyper-BCK-ideal are given.
\end{abstract}

\section{Introduction}

A hypergroup, as a generalization of a group, was introduced by Marty [1] in 1934. Many authors have developed the discussion of hyperstructures (also called multialgebras), such as Corsini [2] and Vougiouklis [3]. We can find wellwritten books for the introduction to hyperstructures, e.g., Corsini [2], Corsini and Leoreanu [4], Davvaz [5, 6], Davvaz and Cristea [7], and Schweigert [8]. Another topic, which has roused the interest of several mathematicians, is that one of hyper-BCK-algebra (briefly, $\mathbb{-}$-BCK-algebra), introduced by Jun et al. [9]. $\llbracket-B C K$-algebras represent a natural extension of classical BCK-algebras. In a classical BCK-algebra, the composition of two elements is an element, while in a $\mathbb{M}$-BCK-algebra, the composition of two elements is a set.

As an extension of the classical notion of a set, Zadeh [10], in 1965, proposed fuzzy sets (briefly, FSs) as mathematical model of vagueness where elements belong to a given set to some degree that is typically a number that belongs to the unit interval $[0,1]$. In 1986, this concept has been generalized to intuitionistic fuzzy set (briefly, IFS) theory by adding a nonmembership function by Atanassov [11]. On the other hand, neutrosophy is an almost new branch in pure mathematics which was introduced in 1998 by Smarandache (see $[12,13])$. It is an extension of the classical idea of a set and it is related to IFS theory and intuitionistic logic. Neutrosophic sets (briefly, NSs) are sets whose elements have independent degrees of truth and indeterminate and false memberships in the unit interval $[0,1]$. The natural generalization of NS theory is the approach of MBJ-neutrosophic sets (briefly, MBJ-NSs), introduced by Takallo et al. [14] in 2018, when they generalized the indeterminacy membership function in a NS to an interval-valued membership function. If the interval-valued indeterminacy membership function of an MBJ-NS takes equal lower and upper values, then we go back to the NS. Thus, MBJ-NSs provide a more adequate description of uncertainty than NSs. Different extensions of FSs have been extensively implemented to algebraic structures, decisionmaking problems, etc. For algebraic structures (especially, $\mathrm{BCK} / \mathrm{BCI}$-algebras and semigroups), see [15-21], and for decision-making problems, see [22-24]. In [14], Takallo et al. 
introduced the concept of an MBJ-N subalgebra as a generalization of a neutrosophic subalgebra in BCK/BCI-algebras and next Jun and Roh [25] introduced and studied the concept of an MBJ-N ideal. In B-algebras, Manokaran and Prakasam [26] introduced the MBJ-N subalgebra and Khalid et al. [27] defined and studied the MBJ-N T-ideal. As a new idea and based on MBJ-NSs, Bordbar et al. [28] proposed the notion of a BMBJ-N subalgebra and Takallo et al. [29] introduced the notions of a BMBJ-N ${ }^{\circ}$-subalgebra and a (closed) BMBJ-N ideal in BCK/BCI-algebras. Borzooei et al. [30] presented a positive implicative BMBJ-N ideal in BCKalgebras.

In an algebraic hyperstructure, Jun and Xin [31] first

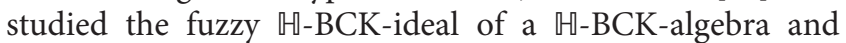
Bakhshi et al. [32] introduced fuzzy (weak, positive) im-

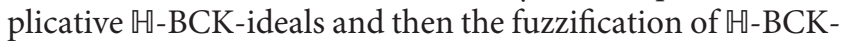
algebra started to grow up. In particular, a link between hyper $\mathbb{-}$-BCK-algebras and IFSs has been established by Borzooei and Jun [33] where they discussed intuitionistic

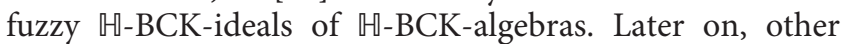
researchers considered this field of study such as Jun [34]

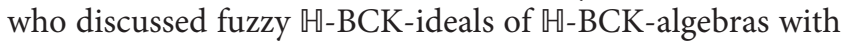
multivalued membership functions. Also, an article was written by Seo et al. on $\mathbb{H}-\mathrm{BCK}$-algebras and multipolar

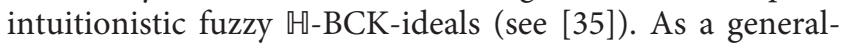
ization of fuzzification of hyperalgebraic structures, some researchers started working on fuzzification of hyperalgebraic structures. In fact, a link between NSs and hyperalgebraic structures was recently established and some work was done in this regard, see [36-39].

In our paper, we combine the notion of $\llbracket$-BCK-algebras with MBJ-NSs to define some types of BMBJ-neutrosophic

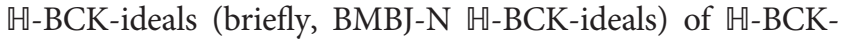
algebras and it is organized as follows: after an introduction, Section 2 briefly reviews some preliminary results related to M-BCK-algebras and MBJ-NSs that are used throughout the paper. Section 3 defines the notions of BMBJ-neutrosophic weak hyper-BCK-ideals (briefly, BMBJ-N WW-BCK-ideals), BMBJ-neutrosophic $s$-weak hyper-BCK-ideals (briefly, BMBJ-N $s$-WM-BCK-ideals), and BMBJ-neutrosophic strong

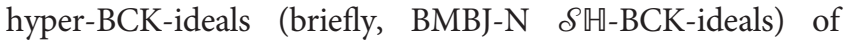
$\because-B C K$-algebras and presents several results related to the new defined concepts. Also, we discuss BMBJ-N WM-BCKideal and BMBJ-N $\mathcal{S}$ 凹-BCK-ideal in relation to level cut sets. We find conditions for a BMBJ-N WW-BCK-ideal to be a BMBJ-N $s$-WW-BCK-ideal. We give conditions for an MBJNS to be a BMBJ-N $\delta$ 田-BCK-ideal. Finally, in Section 4, we present the conclusion and future works of the study.

\section{Preliminaries}

In the current section, we remember some of the basic notions of $\mathbb{-}$-BCK-algebras and MBJ-NSs which will be very helpful in further study of the paper. Let $\widetilde{\mathscr{H}}$ be a $\mathbb{M}$-BCKalgebra in what follows, unless otherwise stated.

Let $\tilde{\mathscr{H}}$ be a nonempty set and let " $\diamond$ " be a mapping

$$
\diamond: \tilde{\mathscr{H}} \times \tilde{\mathscr{H}} \longrightarrow \mathscr{H}(\tilde{\mathscr{H}}) \backslash\{\phi\}
$$

which is said to be hyperoperation. For any two subsets $\mathscr{K}$ and $\mathscr{F}$, denote by $\mathscr{K} \diamond \mathscr{F}$, the set $\bigcup\{\varrho \diamond \tau \mid \varrho \in \mathscr{K}, \tau \in \mathscr{F}\}$. $W$ shall use $\varrho \diamond \tau$ instead of $\{\varrho\} \diamond \tau, \varrho \diamond\{\tau\}$, or $\{\varrho\} \diamond\{\tau\}$.

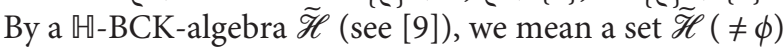
with a special element 0 and a hyperoperation $\diamond$, for all $\varrho, \tau, \eta \in \widetilde{\mathscr{H}}$, that satisfies the following axioms:

(HI) $(\varrho \diamond \eta) \diamond(\tau \diamond \eta)=\varrho \diamond \tau$,

(HII) $(\varrho \diamond \tau) \diamond \eta=(\varrho \diamond \eta) \diamond \tau$,

(HIII) $\varrho \diamond \widetilde{\mathscr{H}} \ll\{\varrho\}$,

(HIV) $\varrho \ll \tau$ and $\tau \ll \varrho$ imply $\varrho=\tau$,

For all $\varrho, \tau, \eta \in \tilde{\mathscr{H}}$, where $\varrho \ll \tau$ is defined by $0 \in \varrho \diamond \tau$ and $\forall \mathscr{K}, \mathscr{F} \subseteq \tilde{\mathscr{H}}, \mathscr{K} \ll \mathscr{F}$ is defined by $\forall r \in \mathscr{K}, \exists t \in \mathscr{F}$ such that $r \ll t$.

In a $\mathbb{H}-\mathrm{BCK}$-algebra $\tilde{\mathscr{H}}$, the axiom (HIII) is equivalent to $(\mathrm{HV})$, where

(HV) $\varrho \diamond \tau \ll\{\varrho\}$ for all $\varrho, \tau \in \tilde{\mathscr{H}}$.

Proposition 1 (see [9]). Every $\mathbb{\sharp}-B C K$-algebra $\tilde{\mathscr{H}}$ satisfies the following conditions, for all $\varrho, \tau, \eta \in \widetilde{\mathscr{H}}$ and for any nonempty subsets $\mathscr{K}, \mathscr{F}, \mathscr{G}$ of $\widetilde{\mathscr{H}}$,

(1) $\varrho \diamond 0 \ll\{\varrho\}, 0 \diamond \varrho=\{0\}, 0 \diamond 0=\{0\}$,

(2) $0 \ll \rho, \varrho \ll \rho, \varrho \in \varrho \diamond 0$,

(3) $\varrho \diamond 0 \ll\{\tau\} \Rightarrow \varrho \ll \tau$,

(4) $\tau \ll \eta \Rightarrow \varrho \diamond \eta \ll \varrho \diamond \tau$,

(5) $\varrho \diamond \tau=\{0\} \Rightarrow \varrho \diamond \eta \ll \tau \diamond \eta$, $(\varrho \diamond \eta) \diamond(\tau \diamond \eta)=\{0\}$,

(6) $\mathscr{K} \subseteq \mathscr{F} \Rightarrow \mathscr{K} \ll \mathscr{F}$,

(7) $\mathscr{K} \ll\{0\} \Rightarrow \mathscr{K}=\{0\}$,

(8) $\mathscr{K} \ll \mathscr{K}$,

$(\mathscr{K} \diamond \mathscr{F}) \diamond \mathscr{G}=(\mathscr{K} \diamond \mathscr{G}) \diamond \mathscr{F}$, $\mathscr{K} \diamond \mathscr{F} \ll \mathscr{K}$,

(9) $\mathscr{K} \diamond\{0\}=\{0\} \Rightarrow \mathscr{K}=\{0\}$.

Definition 1. Let $(\tilde{\mathscr{H}}, \diamond)$ be a $\mathbb{H}-\mathrm{BCK}$-algebra. A subset $\mathscr{K}$ of $\widetilde{\mathscr{H}}$ is called as follows:

A hyper-BCK-ideal (briefly, $\mathbb{-}-$ BCK-ideal) of $\tilde{\mathscr{H}}$ (see [9]) if

(1) $0 \in \mathscr{K}$,

(2) $\varrho \diamond \tau \ll \mathscr{K}, \tau \in \mathscr{K} \Rightarrow \varrho \in \mathscr{K}, \forall \varrho, \tau \tilde{\mathscr{H}}$.

A weak hyper-BCK-ideal (briefly, WWH-BCK-ideal) of $\widetilde{\mathscr{H}}$ (see [9]), if it satisfies (1) and

(3) $\varrho \diamond \tau \subseteq \mathscr{K}, \tau \in \mathscr{K} \Rightarrow \varrho \in \mathscr{K}, \forall \varrho, \tau \in \tilde{\mathscr{H}}$,

A strong hyper-BCK-ideal (briefly, $\mathcal{S} \mathbb{-}$-BCK-ideal) of $\widetilde{\mathscr{H}}$ (see [40]), if it satisfies (1) and

(4) $\varrho \diamond \tau \cap \mathscr{K} \neq \phi, \tau \in \mathscr{K} \Rightarrow \varrho \in \mathscr{K}, \forall \varrho, \tau \in \tilde{\mathscr{H}}$.

By an interval number $\widetilde{\omega}$, we mean an interval $\widetilde{\Phi}=\left[\Phi^{-}, \Phi^{+}\right]$, where $0 \leq \Phi^{-} \leq \Phi^{+} \leq 1$. The set of all closed interval numbers $I$ is denoted by $[I]$. The interval $[\varpi, \emptyset]$ is identified with the number $₫$. 
For two interval numbers $\widetilde{\varpi}_{1}=\left[\Phi_{1}^{-}, \Phi_{1}^{+}\right]$and $\widetilde{\omega}_{2}=\left[\omega_{2}^{-}, \omega_{2}^{+}\right]$, we define

$$
\begin{aligned}
& r \max \left\{\widetilde{\omega}_{1}, \widetilde{\omega}_{2}\right\}=\left[\max \left\{\varpi_{1}^{-}, \omega_{2}^{-}\right\}, \max \left\{\omega_{1}^{+}, \omega_{2}^{+}\right\}\right], \\
& r \min \left\{\widetilde{\omega}_{1}, \widetilde{\omega}_{2}\right\}=\left[\min \left\{\varpi_{1}^{-}, \omega_{2}^{-}\right\}, \min \left\{\varpi_{1}^{+}, \omega_{2}^{+}\right\}\right] \text {, }
\end{aligned}
$$

Furthermore, we have

(1) $\widetilde{\omega}_{1} \pm \widetilde{\Phi}_{2} \Leftrightarrow \Phi_{1}^{-} \geq \Phi_{2}^{-}, \omega_{1}^{+} \geq \Phi_{2}^{+}$,

(2) $\widetilde{\Phi}_{1} \prec \widetilde{\omega}_{2} \Leftrightarrow \Phi_{1}^{-} \leq \Phi_{2}^{-}, \Phi_{1}^{+} \leq \Phi_{2}^{+}$,

(3) $\widetilde{\omega}_{1}=\widetilde{\omega}_{2} \Leftrightarrow \Phi_{1}^{-}=\Phi_{2}^{-}, \omega_{1}^{+}=\Phi_{2}^{+}$.

Let $\tilde{\mathscr{H}}$ be a nonempty set. A function $\widetilde{D}: \tilde{\mathscr{H}} \longrightarrow[I]$ is called an interval-valued fuzzy set (briefly, IVFS) over a universe $\tilde{\mathscr{H}}_{\text {. }}$

Let $[I]^{H}$ stand for the set of all IVFSs $\tilde{\mathscr{H}}$. For any $\widetilde{D} \in[I]^{H}$ and $\varrho \in \tilde{\mathscr{H}}, \widetilde{D}=\left[D^{-}(\varrho), D^{+}(\varrho)\right]$ is called the degree of membership of an element $\varrho$ to $\widetilde{D}$, where $D^{-}(\varrho): \tilde{\mathscr{H}} \longrightarrow I$ and $D^{+}(\varrho): \tilde{\mathscr{H}} \longrightarrow I$ are FSs over a universe $\tilde{\mathscr{H}}$ which are called a lower FS and an upper FS over $\tilde{\mathscr{H}}$, respectively. For simplicity, we denote $\widetilde{D}=\left[D^{-}, D^{+}\right]$.

Let $\tilde{\mathscr{H}}$ be a nonempty set. An NS over a universe $\tilde{\mathscr{H}}$ (see [12]) is a structure of the form:

$$
\mathscr{D}=\left\{\left\langle\varrho ; \mathscr{D}_{T}(\varrho), \mathscr{D}_{I}(\varrho), \mathscr{D}_{F}(\varrho)\right\rangle \mid \varrho \in \tilde{\mathscr{H}}\right\},
$$

where $\mathscr{D}_{T}, \mathscr{D}_{I}$, and $\mathscr{D}_{I}$ are FSs over a universe $\tilde{\mathscr{H}}$, which are called a truth and an indeterminate and false membership functions, respectively.

For the sake of simplicity, we shall use the symbol $\mathscr{D}=$ $\left(\mathscr{D}_{T}, \mathscr{D}_{I}, \mathscr{D}_{F}\right)$ for the NS

$$
\mathscr{D}=\left\{\left\langle\varrho ; \mathscr{D}_{T}(\varrho), \mathscr{D}_{I}(\varrho), \mathscr{D}_{F}(\varrho)\right\rangle \mid \varrho \in \tilde{\mathscr{H}}\right\} .
$$

In [14], Takallo et al. introduced the idea of an MBJ-NS as follows.

Definition 2. Let $\tilde{\mathscr{H}}$ be a nonempty set. By an MBJ-NS over a universe $\tilde{\mathscr{H}}$, we mean a structure of the following form:

$$
\mathscr{W}=\left\{\left\langle\varrho ; \mathscr{M}_{\mathscr{W}}(\varrho), \widetilde{\mathscr{B}}_{\mathscr{W}}(\varrho), \mathscr{J}_{\mathscr{W}}(\varrho)\right\rangle \mid \varrho \in \tilde{\mathscr{H}}\right\},
$$

where $\mathscr{M}_{\mathscr{W}}$ and $\mathscr{J}_{\mathscr{W}}$ are FSs over a universe $\tilde{\mathscr{H}}$, which are called a truth and a false membership functions, respectively, and $\widetilde{\mathscr{B}}_{\mathscr{W}}$ is an interval-valued fuzzy set over a universe $\mathscr{\mathscr { H }}$ which is called an indeterminate interval-valued membership function.

For the sake of simplicity, we shall use the symbol $\mathscr{W}=$ $\left(\mathscr{M}_{\mathscr{W}}, \widetilde{\mathscr{B}}_{\mathscr{W}}, \mathscr{J}_{\mathscr{W}}\right)$ for the MBJ-NS,

$$
\mathscr{W}=\left\{\left\langle\varrho ; \mathscr{M}_{\mathscr{W}}(\varrho), \widetilde{\mathscr{B}}_{\mathscr{W}}(\varrho), \mathscr{J}_{\mathscr{W}}(\varrho)\right\rangle \mid \varrho \in \tilde{\mathscr{H}}\right\}
$$

Given an MBJ-NS $\mathscr{W}=\left(\mathscr{M}_{\mathscr{W}}, \widetilde{\mathscr{B}}_{\mathscr{W}}, \mathscr{J}_{\mathscr{W}}\right)$ over a universe $\tilde{\mathscr{H}}$, we consider the following sets:

$$
\begin{aligned}
U\left(\mathscr{M}_{\mathscr{W}}, \alpha\right) & =\left\{\varrho \in H \mid \mathscr{M}_{\mathscr{W}}(\varrho) \geq \alpha\right\}, \\
L\left(\mathscr{B}_{\mathscr{W}}^{-}, \delta^{-}\right) & =\left\{\varrho \in H \mid \mathscr{B}_{\mathscr{W}}^{-}(\varrho) \leq \delta^{-}\right\}, \\
U\left(\mathscr{B}_{\mathscr{W}}^{+}, \delta^{+}\right) & =\left\{\varrho \in H \mid \mathscr{B}_{\mathscr{W}}^{+}(\varrho) \geq \delta^{+}\right\}, \\
L\left(\mathscr{J}_{\mathscr{W}}, \gamma\right) & =\left\{\varrho \in H \mid \mathscr{J}_{\mathscr{W}}(\varrho) \leq \gamma\right\},
\end{aligned}
$$

where $\alpha, \gamma, \delta^{-}, \delta^{-} \in[0,1]$.

\section{BMBJ-Neutrosophic Hyper-BCK-Ideals}

Definition 3. An MBJ-NS $\mathscr{W}$ on $\widetilde{\mathscr{H}}$ is called a BMBJ-N 凹-BCK-ideal of $\tilde{\mathscr{H}}$, if it satisfies

(1) $(\forall \varrho, \tau \in \tilde{\mathscr{H}})\left(\varrho \ll \tau \Rightarrow\left(\begin{array}{c}\mathscr{M}_{\mathscr{W}}(\varrho) \geq \mathscr{M}_{\mathscr{W}}(\tau) \\ \mathscr{B}_{\mathscr{W}}^{-}(\varrho) \leq \mathscr{B}_{\mathscr{W}}^{-}(\tau) \\ \mathscr{B}_{\mathscr{W}}^{+}(\varrho) \geq \mathscr{B}_{\mathscr{W}}^{+}(\tau) \\ \mathscr{J}_{\mathscr{W}}(\varrho) \leq \mathscr{J}_{\mathscr{W}}(\tau)\end{array}\right)\right)$,

(2) $(\forall \varrho, \tau \in \tilde{\mathscr{H}})$

$\left(\mathscr{M}_{\mathscr{W}}(\varrho) \geq \min \left\{\inf \left\{\mathscr{M}_{\mathscr{W}}(z) \mid z \in \varrho \diamond \tau\right\}, \mathscr{M}_{\mathscr{W}}\right.\right.$

$(\tau)\} \mathscr{B}_{\mathscr{W}}^{-}(\varrho) \leq \max \left\{\sup \left\{\mathscr{B}_{\mathscr{W}}^{-}\right.\right.$

$\left.(z) \mid z \in \varrho \diamond \tau\}, \mathscr{B}_{\mathscr{W}}^{-}(\tau)\right\} \mathscr{B}_{\mathscr{W}}^{+}(\varrho) \geq \min \left\{\inf \left\{\mathscr{B}_{\mathscr{W}}^{+}\right.\right.$

$\left.(z) \mid z \in \varrho \diamond \tau\}, \mathscr{B}_{\mathscr{W}}^{+}(\tau)\right\} \mathscr{J}_{\mathscr{W}}(\varrho) \leq \max \left\{\sup \left\{\mathscr{J}_{\mathscr{W}}\right.\right.$

$\left.\left.(z) \mid z \in \varrho \diamond \tau\}, \mathscr{J}_{\mathscr{W}}(\tau)\right\}\right)$.

Example 1. Let $\tilde{\mathscr{H}}=\{0, \varrho, \tau\}$ be a set with the hyperoperation " $\diamond$ ", which is given by Table 1 .

Then, $\tilde{\mathscr{H}}$ is a $\mathbb{H}$-BCK-algebra. Let $\mathscr{W}=\left(\mathscr{M}_{\mathscr{W}}, \widetilde{\mathscr{B}}_{\mathscr{W}}, \mathscr{J}_{\mathscr{W}}\right)$ be an MBJ-NS over $\tilde{\mathscr{H}}$ given by Table 2 .

It is routine to check that $\mathscr{W}=\left(\mathscr{M}_{\mathscr{W}}, \widetilde{\mathscr{B}}_{\mathscr{W}}, \mathscr{J}_{\mathscr{W}}\right)$ is a BMBJ-N $\mathbb{H}$-BCK-ideal of $\tilde{\mathscr{H}}$.

Proposition 2. Let $\mathscr{W}=\left(\mathscr{M}_{\mathscr{W}}, \widetilde{\mathscr{B}}_{\mathscr{W}}, \mathscr{J}_{\mathscr{W}}\right)$ be a $B M B J-N$ -BCK-ideal of $\tilde{\mathscr{H}}$. Then,

(i) $(\forall \varrho \in \tilde{\mathscr{H}})\left(\begin{array}{c}\mathscr{M}_{\mathscr{W}}(0) \geq \mathscr{M}_{\mathscr{W}}(\varrho) \\ \mathscr{B}_{\mathscr{W}}^{-}(0) \leq \mathscr{B}_{\mathscr{W}}^{-}(\varrho) \\ \mathscr{B}_{\mathscr{W}}^{+}(0) \geq \mathscr{B}_{\mathscr{W}}^{+}(\varrho) \\ \mathscr{J}_{\mathscr{W}}(0) \leq \mathscr{J}_{\mathscr{W}}(\varrho)\end{array}\right)$

(ii) If $\mathscr{W}=\left(\mathscr{M}_{\mathscr{W}}, \widetilde{\mathscr{B}}_{\mathscr{W}}, \mathscr{J}_{\mathscr{W}}\right)$ satisfies

$$
(\forall \mathscr{K} \subseteq \tilde{H})\left(\exists \varrho_{\circ}, \tau_{1}, \tau_{2}, \eta^{\circ} \in \mathscr{K}\right)\left(\begin{array}{c}
\mathscr{M}_{\mathscr{W}}\left(\varrho_{0}\right)=\inf \left\{\mathscr{M}_{\mathscr{W}}(\varrho) \mid \varrho \in \mathscr{K}\right\} \\
\mathscr{B}_{\mathscr{W}}^{-}\left(\tau_{1}\right)=\sup \left\{\mathscr{B}_{\mathscr{W}}^{-}(\tau) \mid \tau \in \mathscr{K}\right\} \\
\mathscr{B}_{\mathscr{W}}^{+}\left(\tau_{2}\right)=\inf \left\{\mathscr{B}_{\mathscr{W}}^{+}(\tau) \mid \tau \in \mathscr{K}\right\} \\
\mathscr{J}_{\mathscr{W}}\left(\eta^{\circ}\right)=\sup \left\{\mathscr{F}_{\mathscr{W}}(\eta) \mid \eta \in \mathscr{K}\right\}
\end{array}\right),
$$


TABLE 1: Tabular representation of the hyperoperation " $\diamond$."

\begin{tabular}{lccc}
\hline$\diamond$ & 0 & $\varrho$ & $\tau$ \\
\hline 0 & $\{0\}$ & $\{0\}$ & $\{0\}$ \\
$\varrho$ & $\{\varrho\}$ & $\{0, \varrho\}$ & $\{0, \varrho\}$ \\
$\tau$ & $\{\tau\}$ & $\{\varrho, \tau\}$ & $\{0, \varrho, \tau\}$ \\
\hline
\end{tabular}

TABLE 2: Tabular representation of $\mathscr{W}=\left(\mathscr{M}_{\mathscr{W}}, \widetilde{\mathscr{B}}_{\mathscr{W}}, \mathscr{J}_{\mathscr{W}}\right)$.

\begin{tabular}{lccc}
\hline $\mathscr{W}$ & $\mathscr{M}_{\mathscr{W}}$ & $\widetilde{\mathscr{B}}_{\mathscr{W}}$ & $\mathcal{J}_{\mathscr{W}}$ \\
\hline 0 & $1 / 5$ & {$[(1 / 4), 0.71]$} & 0.33 \\
$\varrho$ & $1 / 7$ & {$[(1 / 3), 0.51]$} & 0.43 \\
$\tau$ & $1 / 9$ & {$[(1 / 3), 0.21]$} & 0.53 \\
\hline
\end{tabular}

then

$$
(\forall \varrho, \tau \in \tilde{\mathscr{H}})\left(\exists u, v_{1}, v_{2}, w \in \varrho \diamond \tau\right)\left(\begin{array}{c}
\mathscr{M}_{\mathscr{W}}(\varrho) \geq \min \left\{\mathscr{M}_{\mathscr{W}}(u), \mathscr{M}_{\mathscr{W}}(\tau)\right\} \\
\mathscr{B}_{\mathscr{W}}^{-}(\varrho) \leq \max \left\{\mathscr{B}_{\mathscr{W}}^{-}\left(v_{1}\right), \mathscr{B}_{\mathscr{W}}^{-}(\tau)\right\} \\
\mathscr{B}_{\mathscr{W}}^{+}(\varrho) \geq \min \left\{\mathscr{B}_{\mathscr{W}}^{+}\left(v_{2}\right), \mathscr{B}_{\mathscr{W}}^{+}(\tau)\right\} \\
\mathscr{J}_{\mathscr{W}}(\varrho) \leq \max \left\{\mathscr{J}_{\mathscr{W}}(w), \mathscr{M}_{\mathscr{W}}(\tau)\right\}
\end{array}\right) .
$$

Proof. The proof is obvious and is omitted.

Corollary 1. In a finite-BCK-algebra, every $B M B J-N$ $\mathbb{H}$-BCK-ideal $\mathscr{W}=\left(\mathscr{M}_{\mathscr{W}}, \widetilde{\mathscr{B}}_{\mathscr{W}}, \mathscr{J}_{\mathscr{W}}\right)$ over $\tilde{\mathscr{H}}_{\text {satisfies con- }}$ dition (9).

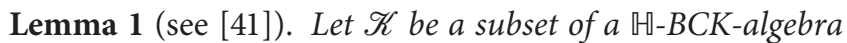
$\tilde{\mathscr{H}}$. If $\mathscr{I}$ is a $\Vdash-B C K$-ideal of $\tilde{\mathscr{H}}$ such that $\mathscr{K} \ll \mathscr{I}$, then $\mathscr{K}$ is contained in $\mathscr{I}$.

Theorem 1. An MBJ-NS $\mathscr{W}=\left(\mathscr{M}_{\mathscr{W}}, \widetilde{\mathscr{B}}_{\mathscr{W}}, \mathscr{J}_{\mathscr{W}}\right)$ over $\tilde{\mathscr{H}}$ is a $B M B J-N \mathbb{M}-B C K$-ideal of $\widetilde{\mathscr{H}}$ if and only if the nonempty sets $U\left(\mathscr{M}_{\mathscr{W}}, \alpha\right), L\left(\mathscr{B}_{\mathscr{V}}^{-}, \delta^{-}\right), U\left(\mathscr{B}_{\mathscr{W}}^{+}, \delta^{+}\right)$, and $L\left(\mathscr{J}_{\mathscr{W}}, \gamma\right)$ are $\mathbb{H}$-BCK-ideals of $\mathscr{H}$ for any $\alpha, \delta^{-}, \delta^{+}, \gamma \in[0,1]$.
Proof. Assume that $\mathscr{W}=\left(\mathscr{M}_{\mathscr{W}}, \widetilde{\mathscr{B}}_{\mathscr{W}}, \mathscr{J}_{\mathscr{W}}\right)$ is a BMBJ-N $\mathbb{H}-\mathrm{BCK}$-ideal of $\widetilde{\mathscr{H}}$. Let $\alpha, \delta^{-}, \delta^{+}, \gamma \in[0,1]$ be such that $U\left(\mathscr{M}_{\mathscr{W}}, \alpha\right), L\left(\mathscr{B}_{\mathscr{W}}^{-}, \delta^{-}\right), U\left(\mathscr{B}_{\mathscr{W}}^{+}, \delta^{+}\right)$, and $L\left(\mathscr{J}_{\mathscr{W}}, \gamma\right)$ are nonempty sets. Clearly, $0 \in U\left(\mathscr{M}_{\mathscr{W}}, \alpha\right), 0 \in L\left(\mathscr{B}_{\mathscr{W}}^{-}, \delta^{-}\right)$, $0 \in U\left(\mathscr{B}_{\mathscr{W}}^{+}, \delta^{+}\right)$, and $0 \in L\left(\mathscr{J}_{\mathscr{W}}, \gamma\right)$ by Proposition 2(i). Let $\varrho, \tau, u_{1}, v_{1}, u_{2}, v_{2}, a, b \in \widetilde{\mathscr{H}}$ be such that $\varrho \diamond \tau \in U\left(\mathscr{M}_{\mathscr{W}}, \alpha\right)$, $\tau \in U\left(\mathscr{M}_{\mathscr{W}}, \alpha\right), \quad u_{1} \diamond v_{1} \in L\left(\mathscr{B}_{\mathscr{W}}^{-}, \delta^{-}\right), \quad v_{1} \in L\left(\mathscr{B}_{\mathscr{W}}^{-}, \delta^{-}\right)$, $u_{2} \diamond v_{2} \in U\left(\mathscr{B}_{\mathscr{W}}^{+}, \delta^{+}\right), v_{2} \in U\left(\mathscr{B}_{\mathscr{W}}^{+}, \delta^{+}\right), a \diamond b \in L\left(\mathscr{J}_{\mathscr{W}}, \gamma\right)$, and $b \in L \quad\left(\mathscr{J}_{\mathscr{W}}, \gamma\right)$. Then, $\quad \varrho \diamond \tau \ll U\left(\mathscr{M}_{\mathscr{W}}, \alpha\right)$, $\tau \in U\left(\mathscr{M}_{\mathscr{W}}, \alpha\right), \quad u_{1} \diamond v_{1} \ll L\left(\mathscr{B}_{\mathscr{W}}^{-}, \delta^{-}\right), \quad v_{1} \in L\left(\mathscr{B}_{\mathscr{W}}^{-}, \delta^{-}\right)$, $u_{2} \diamond v_{2} \ll U\left(\mathscr{B}_{\mathscr{W}}^{+}, \delta^{+}\right), v_{2} \in U\left(\mathscr{B}_{\mathscr{W}}^{+}, \delta^{+}\right), a \diamond b \ll L\left(\mathscr{J}_{\mathscr{W}}, \gamma\right)$, and $b \in L\left(\mathscr{F}_{\mathscr{W}}, \gamma\right)$. It follows that

$(\forall x \in \varrho \diamond \tau)\left(\exists_{x^{\circ}} \in U\left(\mathscr{M}_{\mathscr{W}}, \alpha\right)\right.$ such that $\left.x \ll x^{\circ}\right)$ and so $\mathscr{M}_{\mathscr{W}}(x) \geq \mathscr{M}_{\mathscr{W}}\left(x^{\circ}\right)$,

$\left(\forall y_{1} \in u_{1} \diamond v_{1}\right)\left(\exists y_{1^{\circ}} \in L\left(\mathscr{B}_{\mathscr{W}}^{-}, \delta^{-}\right)\right.$such that $\left.y_{1} \ll y_{1^{\circ}}\right)$ and so $\mathscr{B}_{\mathscr{W}}^{-}\left(y_{1}\right) \leq \mathscr{B}_{\mathscr{W}}^{-}\left(y_{1^{\circ}}\right)$,

$\left(\forall y_{2} \in u_{2} \diamond v_{2}\right)\left(\exists y_{2^{\circ}} \in U\left(\mathscr{B}_{\mathscr{W}}^{+}, \delta^{+}\right)\right.$such that $\left.y_{2} \ll y_{2^{\circ}}\right)$ and so $\mathscr{B}_{\mathscr{W}}^{+}\left(y_{2}\right) \geq \mathscr{B}_{\mathscr{W}}^{+}\left(y_{2^{\circ}}\right)$,

$(\forall z \in a \diamond b)\left(\exists_{z^{\circ}} \in L\left(\mathscr{F}_{\mathscr{W}}, \gamma\right)\right.$ such that $\left.z \ll z^{\circ}\right)$ and so $\mathscr{J}_{\mathscr{W}}(z) \leq \mathscr{J}_{\mathscr{W}}\left(z^{\circ}\right)$,

which imply that $\mathscr{M}_{\mathscr{W}}(x) \geq \alpha, \mathscr{B}_{\mathscr{W}}^{-}\left(y_{1}\right) \leq \delta^{-}, \mathscr{B}_{\mathscr{W}}^{+}\left(y_{2}\right) \geq \delta^{+}$, and $\mathscr{J}_{\mathscr{W}}(z) \leq \gamma$ for all $x \in \varrho \diamond \tau, y_{1} \in u_{1} \diamond v_{1}, y_{2} \in u_{2} \diamond v_{2}$, and $z \in a \diamond b$. Hence, $\inf \left\{\mathscr{M}_{\mathscr{W}}(x) \mid x \in \varrho \diamond \tau\right\} \geq \alpha$, $\sup \left\{\mathscr{B}_{\mathscr{W}}^{-}\left(y_{1}\right) \quad \mid y_{1} \in u_{1} \diamond v_{1}\right\} \leq \delta^{-}, \quad \inf \left\{\mathscr{B}_{\mathscr{W}}^{+}\left(y_{2}\right) \mid y_{2} \in\right.$ $\left.u_{2} \diamond v_{2}\right\} \geq \delta^{+}$, and $\sup \left\{\mathscr{J}_{\mathscr{W}}(z) \mid z \in a \diamond b\right\} \leq \gamma$ and so

$$
\begin{gathered}
\mathscr{M}_{\mathscr{W}}(\varrho) \geq \min \left\{\inf \left\{\mathscr{M}_{\mathscr{W}}(x) \mid x \in \varrho \diamond \tau\right\}, \mathscr{M}_{\mathscr{W}}(\tau)\right\} \geq \min \{\alpha, \alpha\}=\alpha, \\
\mathscr{B}_{\mathscr{W}}^{-}\left(u_{1}\right) \leq \max \left\{\sup \left\{\mathscr{B}_{\mathscr{W}}^{-}\left(y_{1}\right) \mid y_{1} \in u_{1} \diamond v_{1}\right\}, \mathscr{B}_{\mathscr{W}}^{-}\left(v_{1}\right)\right\} \leq \max \left\{\delta^{-}, \delta^{-}\right\}=\delta^{-}, \\
\mathscr{B}_{\mathscr{W}}^{+}\left(u_{2}\right) \geq \min \left\{\inf \left\{\mathscr{B}_{\mathscr{W}}^{+}\left(y_{2}\right) \mid y_{2} \in u_{2} \diamond v_{2}\right\}, \mathscr{B}_{\mathscr{W}}^{+}\left(v_{2}\right)\right\} \geq \min \left\{\delta^{+}, \delta^{+}\right\}=\delta^{+}, \\
\mathscr{J}_{\mathscr{W}}(a) \leq \max \left\{\sup \left\{\mathscr{J}_{\mathscr{W}}(z) \mid z \in a \diamond b\right\}, \mathscr{J}_{\mathscr{W}}(b)\right\} \leq \max \{\gamma, \gamma\}=\gamma .
\end{gathered}
$$


Thus, $\varrho \in U\left(\mathscr{M}_{\mathscr{W}}, \alpha\right), u_{1} \in L\left(\mathscr{B}_{\mathscr{W}}^{-}, \delta^{-}\right), u_{2} \in U\left(\mathscr{B}_{\mathscr{W}}^{+}, \delta^{+}\right)$, and $a \in L\left(\mathscr{F}_{\mathscr{W}}, \gamma\right)$, and hence, $U\left(\mathscr{M}_{\mathscr{W}}, \alpha\right), L\left(\mathscr{B}_{\mathscr{W}}^{-}, \delta^{-}\right)$, $U\left(\mathscr{B}_{\mathscr{W}}^{+}, \delta^{+}\right)$, and $L\left(\mathscr{F}_{\mathscr{W}}, \gamma\right)$ are $\mathbb{H}$-BCK-ideals of $\tilde{\mathscr{H}}$.

Conversely, suppose the nonempty sets $U\left(\mathscr{M}_{\mathscr{W}}, \alpha\right)$, $L\left(\mathscr{B}_{\mathscr{W}}^{-}, \delta^{-}\right), U\left(\mathscr{B}_{\mathscr{W}}^{+}, \delta^{+}\right)$, and $L\left(\mathscr{J}_{\mathscr{W}}, \gamma\right)$ are $\mathbb{U}$-BCK-ideals of $\widetilde{\mathscr{H}}$ for all $\alpha, \delta^{-}, \delta^{+}, \gamma \in[0,1]$. Let $\varrho, \tau, u_{1}, v_{1}, u_{2}, v_{2}, a, b \in \tilde{\mathscr{H}}$ be such that $\varrho \ll \tau, u_{1} \ll v_{1}, u_{2} \ll v_{2}, a \ll b, \mathscr{M}_{\mathscr{W}}(\tau)=\alpha$, $\mathscr{B}_{\mathscr{W}}^{-}\left(v_{1}\right)=\delta^{-}, \mathscr{B}_{\mathscr{W}}^{+}\left(v_{2}\right)=\delta^{+}$, and $\mathscr{J}_{\mathscr{W}}(b)=\gamma$. Then, $\tau \in U\left(\mathscr{M}_{\mathscr{W}}, \alpha\right), \quad v_{1} \in L\left(\mathscr{B}_{\mathscr{W}}^{-}, \delta^{-}\right), \quad v_{2} \in U\left(\mathscr{B}_{\mathscr{W}}^{+}, \delta^{+}\right)$, and $b \in L\left(\mathscr{F}_{\mathscr{W}}, \gamma\right)$, and so $\{\varrho\} \ll U\left(\mathscr{M}_{\mathscr{W}}, \alpha\right),\left\{u_{1}\right\} \ll L\left(\mathscr{B}_{\mathscr{W}}^{-}, \delta^{-}\right)$, $\left\{u_{2}\right\} \ll U\left(\mathscr{B}_{\mathscr{W}}^{+}, \delta^{+}\right)$, and $\{a\} \ll L\left(\mathscr{F}_{\mathscr{W}}, \gamma\right)$. From Lemma 1, we have $\quad\{\varrho\} \subseteq U\left(\mathscr{M}_{\mathscr{W}}, \alpha\right), \quad\left\{u_{1}\right\} \subseteq L\left(\mathscr{B}_{\mathscr{W}}^{-}, \delta^{-}\right)$, $\left\{u_{2}\right\} \subseteq U\left(\mathscr{B}_{\mathscr{W}}^{+}, \delta^{+}\right)$, and $\{a\} \subseteq L\left(\mathscr{F}_{\mathscr{W}}, \gamma\right)$, i.e., $\varrho \in U\left(\mathscr{M}_{\mathscr{W}}, \alpha\right)$, $u_{1} \in L\left(\mathscr{B}_{\mathscr{W}}^{-}, \delta^{-}\right), \quad u_{2} \in U\left(\mathscr{B}_{\mathscr{W}}^{+}, \delta^{+}\right), \quad$ and $a \in L\left(\mathscr{F}_{\mathscr{W}}, \gamma\right)$. Hence, $\mathscr{M}_{\mathscr{W}}(\varrho) \geq \alpha=\mathscr{M}_{\mathscr{W}}(\tau), \mathscr{B}_{\mathscr{W}}^{-}\left(u_{1}\right) \leq \delta^{-}=\mathscr{B}_{\mathscr{W}}^{-}\left(v_{1}\right)$, $\mathscr{B}_{\mathscr{W}}^{+}\left(u_{2}\right) \geq \delta^{+}=\mathscr{B}_{\mathscr{K}}^{+}\left(v_{2}\right)$, and $\mathscr{J}_{\mathscr{W}}(a) \leq \gamma=\mathscr{J}_{\mathscr{W}}(b)$. For any $\varrho, \tau, u, v, a, b \in \mathscr{H}$, let

$$
\begin{aligned}
\alpha & =\min \left\{\inf \left\{\mathscr{M}_{\mathscr{W}}\left(t_{1}\right) \mid t_{1} \in \varrho \diamond \tau\right\}, \mathscr{M}_{\mathscr{W}}(\tau)\right\}, \\
\delta^{-} & =\max \left\{\sup \left\{\mathscr{B}_{\mathscr{W}}^{-}\left(t_{2^{\circ}}\right) \mid t_{2^{\circ}} \in u_{1} \diamond v_{1}\right\}, \mathscr{B}_{\mathscr{W}}^{-}\left(v_{1}\right)\right\}, \\
\delta^{+} & =\min \left\{\inf \left\{\mathscr{B}_{\mathscr{W}}^{+}\left(t_{3^{\circ}}\right) \mid t_{3^{\circ}} \in u_{2} \diamond v_{2}\right\}, \mathscr{B}_{\mathscr{W}}^{+}\left(v_{2}\right)\right\}, \\
\gamma & =\max \left\{\sup \left\{\mathscr{f}_{\mathscr{W}}\left(t_{3}\right) \mid t_{3} \in a \diamond b\right\}, \mathscr{J}_{\mathscr{W}}(b)\right\} .
\end{aligned}
$$

Then, $\tau \in U\left(\mathscr{M}_{\mathscr{W}}, \alpha\right), v_{1} \in L\left(\mathscr{B}_{\mathscr{W}}^{-}, \delta^{-}\right), v_{2} \in U\left(\mathscr{B}_{\mathscr{W}}^{+}, \delta^{+}\right)$, $b \in L\left(\mathscr{F}_{\mathscr{W}}, \gamma\right)$, and

$$
\begin{aligned}
\mathscr{M}_{\mathscr{W}}\left(t_{4}\right) & \geq \inf \left\{\mathscr{M}_{\mathscr{W}}\left(t_{1}\right) \mid t_{1} \in \varrho \diamond \tau\right\} \\
& \geq \min \left\{\inf \left\{\mathscr{M}_{\mathscr{W}}\left(t_{1}\right) \mid t_{1} \in \varrho \diamond \tau\right\}, \mathscr{M}_{\mathscr{W}}(\tau)\right\} \\
& =\alpha, \\
\mathscr{B}_{\mathscr{W}}^{-}\left(t_{5^{\circ}}\right) & \leq \sup \left\{\mathscr{B}_{\mathscr{W}}^{-}\left(t_{2^{\circ}}\right) \mid t_{2^{\circ}} \in u_{1} \diamond v_{1}\right\} \\
& \leq \max \left\{\sup \left\{\mathscr{B}_{\mathscr{W}}^{-}\left(t_{2^{\circ}}\right) \mid t_{2^{\circ}} \in u_{1} \diamond v_{1}\right\}, \mathscr{B}_{\mathscr{W}}^{-}\left(v_{1}\right)\right\} \\
& =\delta^{-}, \\
\mathscr{B}_{\mathscr{W}}^{+}\left(t_{6^{\circ}}\right) & \geq \inf \left\{\mathscr{B}_{\mathscr{W}}^{+}\left(t_{3^{\circ}}\right) \mid t_{3^{\circ}} \in u_{2} \diamond v_{2}\right\} \\
& \geq \min \left\{\inf \left\{\mathscr{B}_{\mathscr{W}}^{+}\left(t_{3^{\circ}}\right) \mid t_{3^{\circ}} \in u_{2} \diamond v_{2}\right\}, \mathscr{B}_{\mathscr{W}}^{+}\left(v_{2}\right)\right\} \\
& =\delta^{+}, \\
\mathscr{J}_{\mathscr{W}}\left(t_{6}\right) & \leq \sup \left\{\mathscr{J}_{\mathscr{W}}\left(t_{3}\right) \mid t_{3} \in a \diamond b\right\} \\
& \leq \max \left\{\sup \left\{\mathscr{J}_{\mathscr{W}}\left(t_{3}\right) \mid t_{3} \in a \diamond b\right\}, \mathscr{J}_{\mathscr{W}}(b)\right\} \\
& =\gamma,
\end{aligned}
$$

for all $t_{4} \in \varrho \diamond \tau, t_{5^{\circ}} \in u_{1} \diamond v_{1}, t_{6^{\circ}} \in u_{2} \diamond v_{2}$, and $t_{6} \in a \diamond b$, i.e, $t_{4} \in U\left(\mathscr{M}_{\mathscr{W}}, \alpha\right), t_{5^{\circ}} \in L\left(\mathscr{B}_{\mathscr{W}}^{-}, \delta^{-}\right), t_{6^{\circ}} \in U\left(\mathscr{B}_{\mathscr{W}}^{+}, \delta^{+}\right)$, and $t_{6} \in L\left(\mathscr{J}_{\mathscr{W}}, \gamma\right)$. Thus, $\quad \varrho \diamond \tau \subseteq U\left(\mathscr{M}_{\mathscr{W}}, \alpha\right), \quad u_{1} \diamond v_{1} \subseteq$ $L\left(\mathscr{B}_{\mathscr{W}}^{-}, \delta^{-}\right), u_{2} \diamond v_{2} \subseteq U\left(\mathscr{B}_{\mathscr{W}}^{+}, \delta^{+}\right)$, and $a \diamond b \subseteq L\left(\mathscr{J}_{\mathscr{W}}, \gamma\right)$, which imply from Proposition 1(6) that $\varrho \diamond \tau \ll U\left(\mathscr{M}_{\mathscr{W}}, \alpha\right)$, $u_{1} \diamond v_{1} \ll L \quad\left(\mathscr{B}_{\mathscr{W}}^{-}, \delta^{-}\right), \quad u_{2} \diamond v_{2} \ll U\left(\mathscr{B}_{\mathscr{W}}^{+}, \delta^{+}\right), \quad$ and $a \diamond b \ll L\left(\mathscr{J}_{\mathscr{W}}, \gamma\right)$. Since $U\left(\mathscr{M}_{\mathscr{W}}, \alpha\right), \quad L\left(\mathscr{B}_{\mathscr{W}}^{-}, \delta^{-}\right)$, $U\left(\mathscr{B}_{\mathscr{W}}^{+}, \delta^{+}\right)$, and $L\left(\mathscr{J}_{\mathscr{W}}, \gamma\right)$ are $\mathbb{H}$-BCK-ideals of $\widetilde{\mathscr{H}}$, we have $\varrho \in U\left(\mathscr{M}_{\mathscr{W}}, \alpha\right), \quad u_{1} \in L\left(\mathscr{B}_{\mathscr{W}}^{-}, \delta^{-}\right), \quad u_{2} \in U\left(\mathscr{B}_{\mathscr{W}}^{+}, \delta^{+}\right)$, and $a \in L\left(\mathscr{J}_{\mathscr{W}}, \gamma\right)$, which imply that

$$
\begin{aligned}
& \mathscr{M}_{\mathscr{W}}(\varrho) \geq \alpha=\min \left\{\inf \left\{\mathscr{M}_{\mathscr{W}}\left(t_{1}\right) \mid t_{1} \in \varrho \diamond \tau\right\}, \mathscr{M}_{\mathscr{W}}(\tau)\right\}, \\
& \mathscr{B}_{\mathscr{W}}^{-}\left(u_{1}\right) \leq \delta^{-}=\max \left\{\sup \left\{\mathscr{B}_{\mathscr{W}}^{-}\left(t_{2^{\circ}}\right) \mid t_{2^{\circ}} \in u_{1} \diamond v_{1}\right\}, \mathscr{B}_{\mathscr{W}}^{-}\left(v_{1}\right)\right\}, \\
& \mathscr{B}_{\mathscr{W}}^{+}\left(u_{2}\right) \geq \delta^{+}=\min \left\{\inf \left\{\mathscr{B}_{\mathscr{W}}^{+}\left(t_{3^{\circ}}\right) \mid t_{3^{\circ}} \in u_{2} \diamond v_{2}\right\}, \mathscr{B}_{\mathscr{W}}^{+}\left(v_{2}\right)\right\}, \\
& \mathscr{J}_{\mathscr{W}}(a) \leq \gamma=\max \left\{\sup \left\{\mathscr{J}_{\mathscr{W}}\left(t_{3}\right) \mid t_{3} \in a \diamond b\right\}, \mathscr{J}_{\mathscr{W}}(b)\right\} .
\end{aligned}
$$

Therefore, $\mathscr{W}=\left(\mathscr{M}_{\mathscr{W}}, \widetilde{\mathscr{B}}_{\mathscr{W}}, \mathscr{J}_{\mathscr{W}}\right)$ is a BMBJ-N $\mathbb{H}-\mathrm{BCK}$ ideal of $\tilde{\mathscr{H}}$.

Definition 4. An MBJ-NS $\mathscr{W}=\left(\mathscr{M}_{\mathscr{W}}, \mathscr{B}_{\mathscr{W}}^{-}, \mathscr{J}_{\mathscr{W}}\right)$ over $\tilde{\mathscr{H}}$ is called as follows:

(1) A BMBJ-N WH-BCK-ideal of $\tilde{\mathscr{H}}$ if it satisfies Proposition 2(i) and Definition 3(2).

(2) A BMBJ-N $s$-WM-BCK-ideal of $\tilde{\mathscr{H}}$ if it satisfies Proposition 2(i) and (2).

Theorem 2. Every $B M B J-N$ ㅁ- $B C K$-ideal is a $B M B J-N$ WП-BCK-ideal.

Proof. Straightforward.

The converse of Theorem 2 is not true in general, as seen in the following example.For the converse of Theorem 3, it is not easy to find an example of a BMBJ-N WW-BCK-ideal which is not a BMBJ-N $s$-WM-BCK-ideal. So, we give the following theorem.

Example 2. Let $\tilde{\mathscr{H}}=\{0, \varrho, \tau\}$ be a $\mathbb{H}-\mathrm{BCK}$-algebra as in Example 1. Let $\mathscr{W}=\left(\mathscr{M}_{\mathscr{W}}, \widetilde{\mathscr{B}}_{\mathscr{W}}, \mathscr{J}_{\mathscr{W}}\right)$ be an MBJ-NS over $\tilde{\mathscr{H}}$ given by Table 3 .

Then, $\mathscr{W}=\left(\mathscr{M}_{\mathscr{W}}, \widetilde{\mathscr{B}}_{\mathscr{W}}, \mathscr{J}_{\mathscr{W}}\right)$ is a BMBJ-N WW-BCKideal of $\widetilde{\mathscr{H}}$. Note that $\varrho \ll \tau$,

$$
\begin{aligned}
& \mathscr{M}_{\mathscr{W}}(\varrho)=0.2<0.5=\mathscr{M}_{\mathscr{W}}(\tau), \\
& \mathscr{B}_{\mathscr{W}}^{-}(\varrho)=0.5>0.3=\mathscr{B}_{\mathscr{W}}^{-}(\tau), \\
& \mathscr{B}_{\mathscr{W}}^{+}(\varrho)=0.55<0.85=\mathscr{B}_{\mathscr{W}}^{+}(\tau), \\
& \mathscr{J}_{\mathscr{W}}(\varrho)=0.6>0.5=\mathscr{J}_{\mathscr{W}}(\tau) .
\end{aligned}
$$

Hence, $\mathscr{W}=\left(\mathscr{M}_{\mathscr{W}}, \widetilde{\mathscr{B}}_{\mathscr{W}}, \mathscr{J}_{\mathscr{W}}\right)$ is not a BMBJ-N $\mathbb{H}-\mathrm{BCK}$ ideal of $\tilde{\mathscr{H}}$.

Theorem 3. In a $\mathbb{H}-B C K$-algebra, every $B M B J-N$ $s$-WM-BCK-ideal is a BMBJ-N WW-BCK-ideal.

Proof. Let $\mathscr{W}=\left(\mathscr{M}_{\mathscr{W}}, \widetilde{\mathscr{B}}_{\mathscr{W}}, \mathscr{J}_{\mathscr{W}}\right)$ be a BMBJ-N $s$-WW-BCK-ideal of over $\widetilde{\mathscr{H}}$ and let $\varrho, \tau, u_{1}, v_{1}, u$ $2, v_{2}, a, b \in \tilde{\mathscr{H}}$. Then, there exist $z_{1} \in \varrho \diamond \tau, z_{2} \in u_{1} \diamond v_{1}$, $z_{3} \in u_{2} \diamond v_{2}$, and $z_{4} \in a \diamond b$ such that $\mathscr{M}_{\mathscr{W}}(\varrho) \geq \min$ $\left\{\mathscr{M}_{\mathscr{W}}\left(z_{1}\right), \mathscr{M}_{\mathscr{W}}(\tau)\right\}, \mathscr{B}_{\mathscr{W}}^{-}\left(u_{1}\right) \leq \max \left\{\mathscr{B}_{\mathscr{W}}^{-}\left(z_{2}\right), \mathscr{B}_{\mathscr{W}}^{-}\left(v_{1}\right)\right\}$, $\mathscr{B}_{\mathscr{W}}^{+}\left(u_{2}\right) \geq \min \left\{\mathscr{B}_{\mathscr{W}}^{+}\left(z_{3}\right), \mathscr{B}_{\mathscr{W}}^{+}\left(v_{2}\right)\right\}$, and $\mathscr{J}_{\mathscr{W}} \quad(a) \leq \max$ 
TABLE 3: Tabular representation of $\mathscr{W}=\left(\mathscr{M}_{\mathscr{W}}, \widetilde{\mathscr{B}}_{\mathscr{W}}, \mathscr{J}_{\mathscr{W}}\right)$.

\begin{tabular}{cccc}
\hline $\mathscr{W}$ & $\mathscr{M}_{\mathscr{W}}$ & $\widetilde{\mathscr{B}}_{\mathscr{W}}$ & $\mathscr{J}_{\mathscr{W}}$ \\
\hline 0 & 0.6 & {$[0.2,0.95]$} & 0.3 \\
$\varrho$ & 0.2 & {$[0.5,0.55]$} & 0.6 \\
$\tau$ & 0.5 & {$[0.3,0.85]$} & 0.5 \\
\hline
\end{tabular}

$\left\{\mathscr{J}_{\mathscr{W}}\left(z_{4}\right), \mathscr{f}_{\mathscr{W}}(b)\right\}$ by the condition (ii) of Proposition 2 . Since $\mathscr{M}_{\mathscr{W}}\left(z_{1}\right) \geq \inf \left\{\mathscr{M}_{\mathscr{W}}\left(z_{2}\right) \mid z_{2} \in \varrho \diamond \tau\right\}, \mathscr{B}_{\mathscr{W}}^{-}\left(z_{2}\right) \leq \sup$ $\left\{\mathscr{B}_{\mathscr{W}}^{-}\left(z_{3}\right) \mid z_{3} \in u_{1} \diamond v_{1}\right\}, \quad \mathscr{B}_{\mathscr{W}}^{+}\left(z_{3}\right) \geq \inf \left\{\mathscr{B}_{\mathscr{W}}^{+}\left(z_{4}\right) \mid z_{4} \in u_{2}\right.$ $\left.\diamond v_{2}\right\}$, and $\mathscr{J}_{\mathscr{W}}\left(z_{4}\right) \leq \sup \left\{\mathscr{J}_{\mathscr{W}}\left(z_{5}\right) \mid z_{5} \in a \diamond b\right\}$, it follows that

$$
\begin{aligned}
\mathscr{M}_{\mathscr{W}}(\varrho) & \geq \min \left\{\inf \left\{\mathscr{M}_{\mathscr{W}}\left(z_{2}\right) \mid z_{2} \in \varrho \diamond \tau\right\}, \mathscr{M}_{\mathscr{W}}(\tau)\right\}, \\
\mathscr{B}_{\mathscr{W}}^{-}\left(u_{1}\right) & \leq \max \left\{\sup \left\{\mathscr{B}_{\mathscr{W}}^{-}\left(z_{3}\right) \mid z_{3} \in u_{1} \diamond v_{1}\right\}, \mathscr{B}_{\mathscr{W}}^{-}\left(v_{1}\right)\right\}, \\
\mathscr{B}_{\mathscr{W}}^{+}\left(u_{2}\right) & \geq \min \left\{\inf \left\{\mathscr{B}_{\mathscr{W}}^{+}\left(z_{4}\right) \mid z_{4} \in u_{2} \diamond v_{2}\right\}, \mathscr{B}_{\mathscr{W}}^{+}\left(v_{2}\right)\right\}, \\
\mathscr{J}_{\mathscr{W}}(a) & \leq \max \left\{\sup \left\{\mathscr{J}_{\mathscr{W}}\left(z_{5}\right) \mid z_{5} \in a \diamond b\right\}, \mathscr{J}_{\mathscr{W}}(b)\right\} .
\end{aligned}
$$

Therefore, $\quad \mathscr{W}=\left(\mathscr{M}_{\mathscr{W}}, \widetilde{\mathscr{B}}_{\mathscr{W}}, \mathscr{F}_{\mathscr{W}}\right)$ is a BMBJ-N WW-BCK-ideal of over $\tilde{\mathscr{H}}$.

Theorem 4. Let $\mathscr{W}=\left(\mathscr{M}_{\mathscr{W}}, \widetilde{\mathscr{B}}_{\mathscr{W}}, \mathscr{J}_{\mathscr{W}}\right)$ be a $B M B J-N$ $\mathbb{W} \because-B C K$-ideal of $\tilde{\mathscr{H}}$ which satisfies condition (1) of Proposition 2. Then, $\mathscr{W}=\left(\mathscr{M}_{\mathscr{W}}, \mathscr{\mathscr { B }}_{\mathscr{W}}, \mathscr{J}_{\mathscr{W}}\right)$ is a $B M B J-N$ $s$-WW-BCK-ideal of $\tilde{\mathscr{H}}$.

Proof. For any $\varrho, \tau, u_{1}, v_{1}, u_{2}, v_{2}, a, b \in \tilde{\mathscr{H}}$, there exist $r^{\circ} \in \varrho \diamond \tau,_{s^{\circ}} \in u_{1} \diamond v_{1}, s_{\circ} \in u_{2} \diamond v_{2}$, and $t_{t^{\circ}} \in a \diamond b$ such that $\mathscr{M}_{\mathscr{W}}\left(r^{\circ}\right)=\inf \left\{\mathscr{M}_{\mathscr{W}}(r) \mid r \in \varrho \diamond \tau\right\}, \mathscr{B}_{\mathscr{W}}^{-}\left(s^{\circ}\right)=\sup \left\{\mathscr{B}_{\mathscr{W}}^{-}(s)\right.$ $\left.\mid s \in u_{1} \diamond v_{1}\right\}, \mathscr{B}_{\mathscr{W}}^{+}\left(s_{\circ}\right)=\inf \left\{\mathscr{B}_{\mathscr{W}}^{+}(s) \mid s \in u_{2} \diamond v_{2}\right\}$, and $\mathscr{J}_{\mathscr{W}}$ $\left(t^{\circ}\right)=\sup \left\{\mathscr{F}_{\mathscr{W}}(t) \mid t \in a \diamond b\right\}$. Then,

$$
\begin{aligned}
\mathscr{M}_{\mathscr{W}}(\varrho) & \geq \min \left\{\inf \left\{\mathscr{M}_{\mathscr{W}}(r) \mid r \in \varrho \diamond \tau\right\}, \mathscr{M}_{\mathscr{W}}(\tau)\right\} \\
& =\min \left\{\mathscr{M}_{\mathscr{W}}\left(r^{\circ}\right), \mathscr{M}_{\mathscr{W}}(\tau)\right\}, \\
\mathscr{B}_{\mathscr{W}}^{-}\left(u_{1}\right) & \leq \max \left\{\sup \left\{\mathscr{B}_{\mathscr{W}}^{-}(s) \mid s \in u_{\diamond} v_{1}\right\}, \mathscr{B}_{\mathscr{W}}^{-}\left(v_{1}\right)\right\} \\
& =\max \left\{\mathscr{B}_{\mathscr{W}}^{-}\left(s^{\circ}\right), \mathscr{B}_{\mathscr{W}}^{-}\left(v_{1}\right)\right\}, \\
\mathscr{B}_{\mathscr{W}}^{+}\left(u_{2}\right) & \geq \min \left\{\inf \left\{\mathscr{B}_{\mathscr{W}}^{+}(s) \mid s \in u_{2} \diamond v_{2}\right\}, \mathscr{B}_{\mathscr{W}}^{+}\left(v_{2}\right)\right\} \\
& =\min \left\{\mathscr{B}_{\mathscr{W}}^{+}\left(s_{\circ}\right), \mathscr{B}_{\mathscr{W}}^{+}\left(v_{2}\right)\right\}, \\
\mathscr{J}_{\mathscr{W}}(a) & \leq \max \left\{\sup \left\{\mathscr{J}_{\mathscr{W}}(t) \mid t \in a \diamond b\right\}, \mathscr{J}_{\mathscr{W}}(b)\right\} \\
& =\max \left\{\mathscr{f}_{\mathscr{W}}\left({ }_{t^{\circ}}\right), \mathscr{J}_{\mathscr{W}}(b)\right\} .
\end{aligned}
$$

Therefore, $\quad \mathscr{W}=\left(\mathscr{M}_{\mathscr{W}}, \widetilde{\mathscr{B}}_{\mathscr{W}}, \mathscr{F}_{\mathscr{W}}\right) \quad$ is a BMBJ-N $s$-WW-BCK-ideal of over $\mathscr{H}$.

Theorem 5. An MBJ-NS $\mathscr{W}=\left(\mathscr{M}_{\mathscr{W}}, \widetilde{\mathscr{B}}_{\mathscr{W}}, \mathscr{J}_{\mathscr{W}}\right)$ over $\tilde{\mathscr{H}}$ is a $B M B J-N$ WU- $B C K$-ideal of $\tilde{\mathscr{H}}$ if and only if the nonempty sets $U\left(\mathscr{M}_{\mathscr{W}}, \alpha\right), L\left(\mathscr{B}_{\mathscr{W}}^{-}, \delta^{-}\right), U\left(\mathscr{B}_{\mathscr{W}}^{+}, \delta^{+}\right)$, and $L\left(\mathscr{J}_{\mathscr{W}}, \gamma\right)$ are WW--BCK-ideals of $\tilde{\mathscr{H}}$ for all $\alpha, \delta^{-}, \delta^{-}, \gamma \in[0,1]$.

Proof. It is similar to the proof of Theorem 1.
The following definition presents the BMBJ-N $\mathcal{S} \square-B C K-$ ideal of a $\mathbb{H}$-BCK-algebra $\tilde{\mathscr{H}}$. Next, we study some properties of this concept.

Definition 5. An MBJ-NS $\mathscr{W}=\left(\mathscr{M}_{\mathscr{W}}, \widetilde{\mathscr{B}}_{\mathscr{W}}, \mathscr{J}_{\mathscr{W}}\right)$ over $\tilde{\mathscr{H}}$ is called a BMBJ-N $\mathcal{S}$ U-B-BCK-ideal of $\tilde{\mathscr{H}}$, if it satisfies

(1) $(\forall \varrho, \tau \in \tilde{\mathscr{H}}) \quad\left(\inf \quad\left\{\mathscr{M}_{\mathscr{W}}\left(z_{1}\right) \mid z_{1} \in \varrho \diamond \varrho\right\} \geq \mathscr{M}_{\mathscr{W}}\right.$ (@) $\mathscr{M}_{\mathscr{W}}(\varrho) \geq \min \left\{\sup \left\{\mathscr{M}_{\mathscr{W}}\left(z_{2}\right) \mid z_{2} \in \varrho \diamond \tau\right\}, \mathscr{M}_{\mathscr{W}}\right.$ $(\tau)\})$,

(2) $\left(\forall u_{1}, v_{1} \in \tilde{\mathscr{H}}\right)\left(\sup \left\{\mathscr{B}_{\mathscr{W}}^{-}\left(z_{2}\right) \mid z_{2} \in u_{1} \diamond u_{1}\right\} \leq \mathscr{B}_{\mathscr{W}}^{-}\right.$ $\left(u_{1}\right) \mathscr{B}_{\mathscr{W}}^{-}\left(u_{1}\right) \leq \max \left\{\inf \quad\left\{\mathscr{B}_{\mathscr{W}}^{-}\left(z_{3}\right) \mid z_{3} \in u_{1} \diamond v_{1}\right\}\right.$, $\left.\left.\mathscr{B}_{\mathscr{W}}^{-}\left(v_{1}\right)\right\}\right)$

(3) $\left(\forall u_{2}, v_{2} \in \tilde{\mathscr{H}}\right) \quad\left(\inf \left\{\mathscr{B}_{\mathscr{W}}^{+}\left(z_{3}\right) \mid z_{3} \in u_{2} \diamond u_{2}\right\} \geq \mathscr{B}_{\mathscr{W}}^{+}\right.$ $\left(u_{2}\right)$

$\mathscr{B}_{\mathscr{W}}^{+}\left(u_{2}\right) \geq \min \left\{\sup \left\{\mathscr{B}_{\mathscr{W}}^{+}\left(z_{4}\right) \mid z_{4} \in u_{2} \diamond v_{2}\right\}, \mathscr{B}_{\mathscr{W}}^{+}\right.$ $\left.\left.\left(v_{2}\right)\right\}\right)$

(4) $(\forall w, z \in \tilde{\mathscr{H}}) \quad\left(\sup \quad\left\{\mathscr{F}_{\mathscr{W}}\left(z_{4}\right) \quad \mid z_{4} \in w \diamond w\right\} \leq \mathscr{J}_{\mathscr{W}}\right.$ $(w) \mathscr{J}_{\mathscr{W}}(w) \leq \max \left\{\inf \left\{\mathscr{J}_{\mathscr{W}}\left(z_{5}\right) \mid z_{5} \in w \diamond z\right\}\right.$, $\left.\left.\mathscr{J}_{\mathscr{W}}(z)\right\}\right)$

Example 3. Let $\tilde{\mathscr{H}}=\{0, \varrho, \tau\}$ be a set with the hyperoperation " $\diamond$," which is given by Table 4 .

Then, $\quad \mathscr{\mathscr { H }}^{\prime}$ is a $\mathbb{U}$-BCK-algebra (see [9]). Let $\mathscr{W}=\left(\mathscr{M}_{\mathscr{W}}, \widetilde{\mathscr{B}}_{\mathscr{W}}, \mathscr{J}_{\mathscr{W}}\right)$ be an MBJ-NS over $\tilde{\mathscr{H}}$ given by Table 5.

It is routine to check that $\mathscr{W}=\left(\mathscr{M}_{\mathscr{W}}, \widetilde{\mathscr{B}}_{\mathscr{W}}, \mathscr{J}_{\mathscr{W}}\right)$ is a BMBJ-N $\mathcal{S} \llbracket$-BCK-ideal of $\tilde{\mathscr{H}}$.

Proposition 3. Every $B M B J-N \quad \mathcal{S} \llbracket-B C K$-ideal $\mathscr{W}=$ $\left(\mathscr{M}_{\mathscr{W}}, \widetilde{\mathscr{B}}_{\mathscr{W}}, \mathscr{F}_{\mathscr{W}}\right)$ over $\tilde{\mathscr{H}}$, satisfying the following

conditions: $(1)(\forall \in \tilde{\mathscr{H}})\left(\begin{array}{c}\mathscr{M}_{\mathscr{W}}(0) \geq \mathscr{M}_{\mathscr{W}}(\varrho), \\ \mathscr{B}_{\mathscr{W}}(0) \leq \mathscr{B}_{\mathscr{W}}(\varrho), \\ \mathscr{B}_{\mathscr{W}}^{+}(0) \geq \mathscr{B}_{\mathscr{W}}^{+}(\varrho), \\ \mathscr{J}_{\mathscr{W}}(0) \leq \mathscr{J}_{\mathscr{W}}(\varrho)\end{array}\right)$,

(2) $(\forall \varrho, \tau \in \widetilde{\mathscr{H}})\left(\varrho \ll \tau \Rightarrow\left(\begin{array}{c}\mathscr{M}_{\mathscr{W}}(\varrho) \geq \mathscr{M}_{\mathscr{W}}(\tau), \\ \mathscr{B}_{\mathscr{W}}(\varrho) \leq \mathscr{B}_{\mathscr{W}}^{-}(\tau), \\ \mathscr{B}_{\mathscr{W}}^{+}(\varrho) \geq \mathscr{B}_{\mathscr{W}}^{+}(\tau), \\ \mathscr{f}_{\mathscr{W}}(\varrho) \leq \mathscr{J}_{\mathscr{W}}(\tau)\end{array}\right)\right)$,

(3) $(\forall z, \varrho, \tau \in \tilde{\mathscr{H}})(z \in \varrho \diamond \tau \Rightarrow$

$$
\left.\left(\begin{array}{c}
\mathscr{M}_{\mathscr{W}}(\varrho) \geq \min \left\{\mathscr{M}_{\mathscr{W}}(z), \mathscr{M}_{\mathscr{W}}(\tau)\right\}, \\
\mathscr{B}_{\mathscr{W}}^{-}(\varrho) \leq \max \left\{\mathscr{B}_{\mathscr{W}}(z), \mathscr{B}_{\mathscr{W}}^{-}(\tau)\right\}, \\
\mathscr{B}_{\mathscr{W}}^{+}(\varrho) \geq \min \left\{\mathscr{B}_{\mathscr{W}}^{+}(z), \mathscr{B}_{\mathscr{W}}^{+}(\tau)\right\}, \\
\mathscr{J}_{\mathscr{W}}(\varrho) \leq \max \left\{\mathscr{J}_{\mathscr{W}}(z), \mathscr{J}_{\mathscr{W}}(\tau)\right\}
\end{array}\right)\right) .
$$

Proof. (1) Since $0 \in \varrho \diamond \varrho \forall \varrho \in \tilde{\mathscr{H}}$, we have

$$
\begin{aligned}
& \mathscr{M}_{\mathscr{W}}(0) \geq \inf \left\{\mathscr{M}_{\mathscr{W}}\left(z_{1}\right) \mid z_{1} \in \varrho \diamond \varrho\right\} \geq \mathscr{M}_{\mathscr{W}}(\varrho), \\
& \mathscr{B}_{\mathscr{W}}^{-}(0) \leq \sup \left\{\mathscr{B}_{\mathscr{W}}^{-}\left(z_{2}\right) \mid z_{2} \in \varrho \diamond \varrho\right\} \leq \mathscr{B}_{\mathscr{W}}^{-}(\varrho), \\
& \mathscr{B}_{\mathscr{W}}^{+}(0) \geq \inf \left\{\mathscr{B}_{\mathscr{W}}^{+}\left(z_{3}\right) \mid z_{3} \in \varrho \diamond \varrho\right\} \geq \mathscr{B}_{\mathscr{W}}^{+}(\varrho), \\
& \mathscr{J}_{\mathscr{W}}(0) \leq \sup \left\{\mathscr{J}_{\mathscr{W}}\left(z_{4}\right) \mid z_{4} \in \varrho \diamond \varrho\right\} \leq \mathscr{J}_{\mathscr{W}}(\varrho),
\end{aligned}
$$

for all $\varrho \in \tilde{\mathscr{H}}$.

(2) Let $\varrho, \tau \in \tilde{\mathscr{H}}$ be such that $\varrho \ll \tau$. Then, $0 \in \varrho \diamond \tau$ and thus $\sup \left\{\mathscr{M}_{\mathscr{W}}\left(z_{1}\right) \mid z_{1} \in \varrho \diamond \tau\right\} \geq \mathscr{M}_{\mathscr{W}}(0), \quad \inf \left\{\mathscr{B}_{\mathscr{W}}^{-}\left(z_{2}\right) \mid z_{2}\right.$ $\epsilon \varrho \diamond \tau\} \leq \mathscr{B}_{\mathscr{W}}^{-}(0), \sup \left\{\mathscr{B}_{\mathscr{W}}^{+}\left(z_{3}\right) \mid z_{3} \in \varrho \diamond \tau\right\} \geq \mathscr{B}_{\mathscr{W}}^{+}(0)$, and 
TABLE 4: Tabular representation of the hyperoperation “ $\diamond$."

\begin{tabular}{lccc}
\hline$\diamond$ & 0 & $\varrho$ & $\tau$ \\
\hline 0 & $\{0\}$ & $\{0\}$ & $\{0\}$ \\
$\varrho$ & $\{\varrho\}$ & $\{0\}$ & $\{\varrho\}$ \\
$\tau$ & $\{\tau\}$ & $\{\tau\}$ & $\{0, \tau\}$ \\
\hline
\end{tabular}

TABLE 5: Tabular representation of $\mathscr{W}=\left(\mathscr{M}_{\mathscr{W}}, \mathscr{B}_{\mathscr{W}}^{-}, \mathscr{J}_{\mathscr{W}}\right)$.

\begin{tabular}{llcl}
\hline $\mathscr{W}$ & $\mathscr{M}_{\mathscr{W}}$ & $\widetilde{\mathscr{B}}_{\mathscr{W}}$ & $\mathcal{J}_{\mathscr{W}}$ \\
\hline 0 & 0.63 & {$[0.09,0.75]$} & 0.30 \\
$\varrho$ & 0.43 & {$[0.17,0.57]$} & 0.50 \\
$\tau$ & 0.32 & {$[0.29,0.37]$} & 0.70 \\
\hline
\end{tabular}

$\inf \left\{\mathscr{J}_{\mathscr{W}}\left(z_{4}\right) \mid z_{4} \in \varrho \diamond \tau\right\} \leq \mathscr{J}_{\mathscr{W}}(0)$. It follows from Defini- $\quad$ for all $z, \varrho, \tau \in \tilde{\mathscr{H}}$ with $z \in \varrho \diamond \tau$.

tion 5 that

$$
\begin{aligned}
\mathscr{M}_{\mathscr{W}}(\varrho) & \geq \min \left\{\sup \left\{\mathscr{M}_{\mathscr{W}}\left(z_{1}\right) \mid z_{1} \in \varrho \diamond \tau\right\}, \mathscr{M}_{\mathscr{W}}(\tau)\right\} \\
& \geq \min \left\{\mathscr{M}_{\mathscr{W}}(0), \mathscr{M}_{\mathscr{W}}(\tau)\right\} \\
& =\mathscr{M}_{\mathscr{W}}(\tau), \\
\mathscr{B}_{\mathscr{W}}^{-}(\varrho) & \leq \max \left\{\inf \left\{\mathscr{B}_{\mathscr{W}}^{-}\left(z_{2}\right) \mid z_{2} \in \varrho \diamond \tau\right\}, \mathscr{B}_{\mathscr{W}}^{-}(\tau)\right\} \\
& \leq \max \left\{\mathscr{B}_{\mathscr{W}}^{-}(0), \mathscr{B}_{\mathscr{W}}^{-}(\tau)\right\} \\
& =\mathscr{B}_{\mathscr{W}}^{-}(\tau), \\
\mathscr{B}_{\mathscr{W}}^{+}(\varrho) & \geq \min \left\{\sup \left\{\mathscr{B}_{\mathscr{W}}^{+}\left(z_{3}\right) \mid z_{3} \in \varrho \diamond \tau\right\}, \mathscr{B}_{\mathscr{W}}^{+}(\tau)\right\} \\
& \geq \min \left\{\mathscr{B}_{\mathscr{W}}^{+}(0), \mathscr{B}_{\mathscr{W}}^{+}(\tau)\right\} \\
& =\mathscr{B}_{\mathscr{W}}^{+}(\tau), \\
\mathscr{J}_{\mathscr{W}}(\varrho) & \leq \max \left\{\inf \left\{\mathscr{J}_{\mathscr{W}}\left(z_{4}\right) \mid z_{4} \in \varrho \diamond \tau\right\}, \mathscr{J}_{\mathscr{W}}(\tau)\right\} \\
& \leq \min \left\{\mathscr{J}_{\mathscr{W}}(0), \mathscr{J}_{\mathscr{W}}(\tau)\right\} \\
& =\mathscr{J}_{\mathscr{W}}(\tau),
\end{aligned}
$$

Corollary 2. If $\mathscr{W}=\left(\mathscr{M}_{\mathscr{W}}, \widetilde{\mathscr{B}}_{\mathscr{W}}, \mathscr{J}_{\mathscr{W}}\right)$ is a $B M B J-N$ $\mathcal{S} \amalg-B C K$-ideal over $\tilde{\mathscr{H}}$, then condition (2) of Definition 3 is valid.

Proof. Note that $\mathscr{M}_{\mathscr{W}}(z) \geq \inf \left\{\mathscr{M}_{\mathscr{W}}(z) \mid z \in \varrho \diamond \tau\right\}$, $\mathscr{B}_{\mathscr{W}}^{-}(z) \leq \sup \left\{\mathscr{B}_{\mathscr{W}}^{-}(z) \mid z \in \varrho \diamond \tau\right\}$,

$\mathscr{B}_{\mathscr{W}}^{+}(z) \geq \inf \left\{\mathscr{B}_{\mathscr{W}}^{+}(z) \mid z \in \varrho \diamond \tau\right\}$, and $\mathscr{J}_{\mathscr{W}}(z) \leq \sup \left\{\mathscr{M}_{\mathscr{W}}\right.$ $(z) \mid z \in \varrho \diamond \tau\}$ for all $z, \varrho, \tau \in \widetilde{\mathscr{H}}$ with $z \in \varrho \diamond \tau$. Hence, condition (2) of Definition 3 follows from Proposition $3(2)$.

Theorem 6. Every $B M B J-N$ SH-BCK-ideal is a $B M B J-N$ M-BCK-ideal.

Proof. Straightforward.

The converse of Theorem 6 is not true in general. That is, i.e., $\quad \mathscr{M}_{\mathscr{W}}(\varrho) \geq \mathscr{M}_{\mathscr{W}}(\tau), \quad \mathscr{B}_{\mathscr{W}}^{-}(\varrho) \leq \mathscr{B}_{\mathscr{W}}^{-}(\tau), \quad \mathscr{B}_{\mathscr{W}}^{+}(\varrho) \geq \mathscr{B}_{\mathscr{W}}^{+}$ $(\tau)$, and $\mathscr{J}_{\mathscr{W}}(\varrho) \leq \mathscr{J}_{\mathscr{W}}(\tau)$ for all $\varrho, \tau \in \widetilde{\mathscr{H}}$ with $\varrho \ll \tau$.

(3) Let $z, \varrho, \tau \in \mathscr{\mathscr { H }}$ be such that $z \in \varrho \diamond \tau$. Then,

$$
\begin{aligned}
\mathscr{M}_{\mathscr{W}}(\varrho) & \geq \min \left\{\sup \left\{\mathscr{M}_{\mathscr{W}}\left(z_{1}\right) \mid z_{1} \in \varrho \diamond \tau\right\}, \mathscr{M}_{\mathscr{W}}(\tau)\right\} \\
& \geq \min \left\{\mathscr{M}_{\mathscr{W}}(z), \mathscr{M}_{\mathscr{W}}(\tau)\right\}, \\
\mathscr{B}_{\mathscr{W}}^{-}(\varrho) & \leq \max \left\{\inf \left\{\mathscr{B}_{\mathscr{W}}^{-}\left(z_{2}\right) \mid z_{2} \in \varrho \diamond \tau\right\}, \mathscr{B}_{\mathscr{W}}^{-}(\tau)\right\} \\
& \leq \max \left\{\mathscr{B}_{\mathscr{W}}^{-}(z), \mathscr{B}_{\mathscr{W}}^{-}(\tau)\right\}, \\
\mathscr{B}_{\mathscr{W}}^{+}(\varrho) & \geq \min \left\{\sup \left\{\mathscr{B}_{\mathscr{W}}^{+}\left(z_{3}\right) \mid z_{3} \in \varrho \diamond \tau\right\}, \mathscr{B}_{\mathscr{W}}^{+}(\tau)\right\} \\
& \geq \min \left\{\mathscr{B}_{\mathscr{W}}^{+}(z), \mathscr{B}_{\mathscr{W}}^{+}(\tau)\right\}, \\
\mathscr{J}_{\mathscr{W}}(\varrho) & \leq \max \left\{\inf \left\{\mathscr{J}_{\mathscr{W}}\left(z_{4}\right) \mid z_{4} \in \varrho \diamond \tau\right\}, \mathscr{J}_{\mathscr{W}}(\tau)\right\} \\
& \leq \max \left\{\mathscr{J}_{\mathscr{W}}(z), \mathscr{J}_{\mathscr{W}}(\tau)\right\},
\end{aligned}
$$
a BMBJ-N $\mathbb{H}-\mathrm{BCK}$-ideal may not be a 7BMBJ-N $\mathcal{S} \llbracket-B C K-$ ideal.

Example 4. Let $\tilde{\mathscr{H}}=\{0, \varrho, \tau\}$ be a hyper-BCK-algebra as in Example 1. Let $\mathscr{W}=\left(\mathscr{M}_{\mathscr{W}}, \widetilde{\mathscr{B}}_{\mathscr{W}}, \mathscr{J}_{\mathscr{W}}\right)$ be an MBJ-NS over $\widetilde{\mathscr{H}}$ given by Table 6 .

Then, $\mathscr{W}=\left(\mathscr{M}_{\mathscr{W}}, \widetilde{\mathscr{B}}_{\mathscr{W}}, \mathscr{J}_{\mathscr{W}}\right)$ is a BMBJ-N $\mathbb{H}$-BCK-ideal of $\tilde{\mathscr{H}}$, but it is not a BMBJ-N $\mathcal{S}$ H-BCK-ideal of $\tilde{\mathscr{H}}$, since

$$
\begin{aligned}
& \mathscr{M}_{\mathscr{W}}(\tau)=0.32<0.63=\mathscr{M}_{\mathscr{W}}(\varrho)=\min \left\{\sup \left\{\mathscr{M}_{\mathscr{W}}(z) \mid z \in \tau \diamond \varrho\right\}, \mathscr{M}_{\mathscr{W}}(\varrho)\right\}, \\
& \mathscr{B}_{\mathscr{W}}^{-}(\tau)=0.19>0.12=\mathscr{B}_{\mathscr{W}}^{-}(\varrho)=\max \left\{\inf \left\{\mathscr{B}_{\mathscr{W}}^{-}(z) \mid z \in \tau \diamond \varrho\right\}, \mathscr{B}_{\mathscr{W}}^{-}(\varrho)\right\}, \\
& \mathscr{B}_{\mathscr{W}}^{+}(\tau)=0.21<0.51=\mathscr{B}_{\mathscr{W}}^{+}(\varrho)=\min \left\{\sup \left\{\mathscr{B}_{\mathscr{W}}^{-}(z) \mid z \in \tau \diamond \varrho\right\}, \mathscr{B}_{\mathscr{W}}^{-}(\varrho)\right\}, \\
& \mathscr{J}_{\mathscr{W}}(\tau)=0.39>0.32=\mathscr{J}_{\mathscr{W}}(\varrho)=\max \left\{\inf \left\{\mathscr{J}_{\mathscr{W}}(z) \mid z \in \tau \diamond \varrho\right\}, \mathscr{J}_{\mathscr{W}}(\varrho)\right\} .
\end{aligned}
$$


TABLe 6: Tabular representation of $\mathscr{W}=\left(\mathscr{M}_{\mathscr{W}}, \widetilde{\mathscr{B}}_{\mathscr{W}}, \mathscr{J}_{\mathscr{W}}\right)$.

\begin{tabular}{lccc}
\hline $\mathscr{W}$ & $\mathscr{M}_{\mathscr{W}}$ & $\widetilde{\mathscr{B}}_{\mathscr{W}}$ & $\mathscr{J}_{\mathscr{W}}$ \\
\hline 0 & 0.63 & {$[0.10,0.71]$} & 0.21 \\
$\varrho$ & 0.63 & {$[0.12,0.51]$} & 0.32 \\
$\tau$ & 0.32 & {$[0.19,0.21]$} & 0.39 \\
\hline
\end{tabular}

Theorem 7. Let $\mathscr{W}=\left(\mathscr{M}_{\mathscr{W}}, \widetilde{\mathscr{B}}_{\mathscr{W}}, \mathscr{J}_{\mathscr{W}}\right)$ be an MBJ-NS over $\widetilde{\mathscr{H}}$. If $\mathscr{W}=\left(\mathscr{M}_{\mathscr{W}}, \widetilde{\mathscr{B}}_{\mathscr{W}}, \mathscr{J}_{\mathscr{W}}\right)$ is a BMBJ-N $\mathcal{S} \mathbb{H}-B C K$-ideal of $\widetilde{\mathscr{H}}$, then the nonempty sets $U\left(\mathscr{M}_{\mathscr{W}}, \alpha\right), L\left(\mathscr{B}_{\mathscr{W}}^{-}, \delta^{-}\right)$, $U\left(\mathscr{B}_{\mathscr{W}}^{+}, \delta^{+}\right)$, and $L\left(\mathscr{J}_{\mathscr{W}}, \gamma\right)$ are $\mathcal{S} \amalg-B C K$-ideals of $\widetilde{\mathscr{H}}$ for all $\alpha, \delta^{-}, \delta^{+}, \gamma \in[0,1]$.

Proof. Assume that $\mathscr{W}=\left(\mathscr{M}_{\mathscr{W}}, \widetilde{\mathscr{B}}_{\mathscr{W}}, \mathscr{J}_{\mathscr{W}}\right)$ is an MBJ-NS over $\tilde{\mathscr{H}}$. Let $\alpha, \delta^{-}, \delta^{+}, \gamma \in[0,1]$ be such that $U\left(\mathscr{M}_{\mathscr{W}}, \alpha\right)$, $L\left(\mathscr{B}_{\mathscr{W}}^{-}, \delta^{-}\right), U\left(\mathscr{B}_{\mathscr{W}}^{+}, \delta^{+}\right)$, and $L\left(\mathscr{J}_{\mathscr{W}}, \gamma\right)$ are nonempty sets. Then, there exist $r \in U\left(\mathscr{M}_{\mathscr{W}}, \alpha\right), \quad t_{1} \in L\left(\mathscr{B}_{\mathscr{W}}^{-}, \delta^{-}\right)$, $t_{2} \in U\left(\mathscr{B}_{\mathscr{W}}^{+}, \delta^{+}\right)$, and $s \in L\left(\mathscr{J}_{\mathscr{W}}, \gamma\right)$, and so $\mathscr{M}_{\mathscr{W}}(r) \geq \alpha$, $\mathscr{B}_{\mathscr{W}}^{-}\left(t_{1}\right) \leq \delta^{-}, \quad \mathscr{B}_{\mathscr{W}}^{+}\left(t_{2}\right) \geq \delta^{+}$, and $\mathscr{J}_{\mathscr{W}}(s) \leq \gamma$. Clearly, $0 \in U\left(\mathscr{M}_{\mathscr{W}}, \alpha\right), \quad 0 \in L\left(\mathscr{B}_{\mathscr{W}}^{-}, \delta^{-}\right), \quad 0 \in U\left(\mathscr{B}_{\mathscr{W}}^{+}, \delta^{+}\right)$, and $0 \in L\left(\mathscr{J}_{\mathscr{W}}, \gamma\right)$ by Proposition 3(1). Now, let $\varrho, \tau, u_{1}, v_{1}, u_{2}, v_{2}, a, b \in \widetilde{\mathscr{H}}$ be such that $\tau \in U\left(\mathscr{M}_{\mathscr{W}}, \alpha\right)$, $(\varrho \diamond \tau) \cap U\left(\mathscr{M}_{\mathscr{W}}, \alpha\right) \neq \phi, \quad v_{1} \in L \quad\left(\mathscr{B}_{\mathscr{W}}^{-}, \delta^{-}\right), \quad\left(u_{1} \diamond v_{1}\right) \cap L$ $\left(\mathscr{B}_{\mathscr{W}}^{-}, \delta^{-}\right) \neq \phi, v_{2} \in U\left(\mathscr{B}_{\mathscr{W}}^{+}, \delta^{+}\right),\left(u_{2} \diamond v_{2}\right) \cap L\left(\mathscr{B}_{\mathscr{W}}^{+}, \delta^{+}\right) \neq \phi$, $b \in L\left(\mathscr{J}_{\mathscr{W}}, \gamma\right)$, and $(a \diamond b) \cap L\left(\mathscr{J}_{\mathscr{W}}, \gamma\right) \neq \phi$. Then, there exist $r^{\circ} \in(\varrho \diamond \tau) \cap U\left(\mathscr{M}_{\mathscr{W}}, \alpha\right), \quad t^{\circ} \in\left(\mathrm{u}_{1} \diamond \mathrm{v}_{1}\right) \cap \mathrm{L}\left(\mathscr{B}_{\mathscr{W}}^{-}, \delta^{-}\right)$, $t_{\circ} \in\left(u_{2} \diamond v_{2}\right) \cap U\left(\mathscr{B}_{\mathscr{W}}^{+}, \delta^{+}\right)$, and $s^{\circ} \in(a \diamond b) \cap L\left(\mathscr{J}_{\mathscr{W}}, \gamma\right)$. Hence, $\quad \mathscr{M}_{\mathscr{W}}\left(r^{\circ}\right) \geq \alpha, \quad \mathscr{B}_{\mathscr{W}}^{-}\left(t^{\circ}\right) \leq \delta^{-}, \quad \mathscr{B}_{\mathscr{W}}^{+}\left(t_{\mathrm{o}}\right) \geq \delta^{+}, \quad$ and $\mathscr{J}_{\mathscr{W}}\left(s^{\circ}\right) \leq \gamma$. It follows that

$$
(\forall \mathscr{K} \subseteq \tilde{\mathscr{H}})\left(\exists \varrho_{\circ}, \tau^{\circ}, \xi^{\circ}, \eta^{\circ} \in \mathscr{K}\right) \operatorname{such} \text { that }\left(\begin{array}{c}
\mathscr{M}_{\mathscr{W}}\left(\varrho_{\circ}\right)=\sup \left\{\mathscr{M}_{\mathscr{W}}(\varrho) \mid \varrho \in \mathscr{K}\right\} \\
\mathscr{B}_{\mathscr{W}}^{-}\left(\tau^{\circ}\right)=\inf \left\{\mathscr{B}_{\mathscr{W}}^{-}(\tau) \mid \tau \in \mathscr{K}\right\} \\
\mathscr{B}_{\mathscr{W}}^{+}\left(\xi^{\circ}\right)=\sup \left\{\mathscr{B}_{\mathscr{W}}^{+}(\xi) \mid \tau \in \mathscr{K}\right\} \\
\mathscr{J}_{\mathscr{W}}\left(\eta^{\circ}\right)=\inf \left\{\mathscr{J}_{\mathscr{W}}(\eta) \mid \eta \in \mathscr{K}\right\}
\end{array}\right) .
$$
$\alpha, \delta^{-}, \delta^{+}, \gamma \in[0,1]$, then $\mathscr{W}=\left(\mathscr{M}_{\mathscr{W}}, \widetilde{\mathscr{B}}_{\mathscr{W}}, \mathscr{J}_{\mathscr{W}}\right)$ is a BMBJ-N $\mathcal{S} \amalg-B C K$-ideal of $\tilde{\mathscr{H}}$.

Proof. Suppose the nonempty sets $U\left(\mathscr{M}_{\mathscr{W}}, \alpha\right), L\left(\mathscr{B}_{\mathscr{W}}^{-}, \delta^{-}\right)$, $U\left(\mathscr{B}_{\mathscr{W}}^{+}, \delta^{+}\right)$, and $L\left(\mathscr{J}_{\mathscr{W}}, \gamma\right)$ are $\delta \uplus-B C K-i d e a l s$ of $\widetilde{\mathscr{H}}^{-}$for all $\alpha, \delta^{-}, \delta^{+}, \gamma \in[0,1]$. Then, $\varrho \in U\left(\mathscr{M}_{\mathscr{W}}, \alpha\right), \tau \in L\left(\mathscr{B}_{\mathscr{W}}^{-}, \delta^{-}\right)$, $\xi \in U\left(\mathscr{B}_{\mathscr{W}}^{+}, \delta^{+}\right)$, and $\eta \in L\left(\mathscr{J}_{\mathscr{W}}, \gamma\right)$ for some $\varrho, \tau, \xi, \eta \in \widetilde{\mathscr{H}}$, and so $\varrho \diamond \varrho \ll\{\varrho\} \subseteq U\left(\mathscr{M}_{\mathscr{W}}, \alpha\right), \tau \diamond \tau \ll\{\tau\} \subseteq L\left(\mathscr{B}_{\mathscr{W}}^{-}, \delta^{-}\right)$, $\xi \diamond \xi \ll\{\xi\} \subseteq U\left(\mathscr{B}_{\mathscr{W}}^{+}, \delta^{+}\right)$, and $\eta \diamond \eta \ll\{\eta\} \subseteq L\left(\mathscr{J}_{\mathscr{W}}, \gamma\right)$. By Lemma 1, we have $\varrho \diamond \varrho \subseteq U\left(\mathscr{M}_{\mathscr{W}}, \alpha\right), \tau \diamond \tau \subseteq L\left(\mathscr{B}_{\mathscr{W}}^{-}, \delta^{-}\right)$, $\xi \diamond \xi \subseteq U\left(\mathscr{B}_{\mathscr{W}}^{+}, \delta^{+}\right)$, and $\eta \diamond \eta \subseteq L\left(\mathscr{J}_{\mathscr{W}}, \gamma\right)$. Thus, for any $a \in \varrho \diamond \varrho, b \in \tau \diamond \tau, \quad c \in \xi \diamond \xi$, and $d \in \eta \diamond \eta$, we get $a \in U\left(\mathscr{M}_{\mathscr{W}}, \alpha\right), b \in L\left(\mathscr{B}_{\mathscr{W}}^{-}, \delta^{-}\right), c \in U\left(\mathscr{B}_{\mathscr{W}}^{+}, \delta^{+}\right), \quad$ and $d \in L\left(\mathscr{J}_{\mathscr{W}}, \gamma\right)$. Hence, $\mathscr{M}_{\mathscr{W}}(a) \geq \alpha, \mathscr{B}_{\mathscr{W}}^{-}(b) \leq \delta^{-}, \mathscr{B}_{\mathscr{W}}^{+}(c)$ $\geq \delta^{+}$, and $\mathscr{J}_{\mathscr{W}}(d) \leq \gamma$. It follows that$$
\inf \left\{\mathscr{M}_{\mathscr{W}}(a) \mid a \in \varrho \diamond \varrho\right\} \geq \alpha=\mathscr{M}_{\mathscr{W}}(\varrho),
$$$$
\sup \left\{\mathscr{B}_{\mathscr{W}}^{-}(b) \mid b \in \tau \diamond \tau\right\} \leq \delta^{-}=\mathscr{B}_{\mathscr{W}}^{-}(\tau) \text {, }
$$$$
\inf \left\{\mathscr{B}_{\mathscr{W}}^{+}(c) \mid c \in \xi \diamond \xi\right\} \geq \delta^{+}=\mathscr{B}_{\mathscr{W}}^{+}(\xi) \text {, }
$$$$
\sup \left\{\mathscr{J}_{\mathscr{W}}(d) \mid d \in \eta \diamond \eta\right\} \leq \gamma=\mathscr{J}_{\mathscr{W}}(\eta) \text {. }
$$

$$
\begin{aligned}
\mathscr{M}_{\mathscr{W}}(\varrho) & \geq \min \left\{\sup \left\{\mathscr{M}_{\mathscr{W}}(r) \mid r \in \varrho \diamond \tau\right\}, \mathscr{M}_{\mathscr{W}}(\tau)\right\} \\
& \geq \min \left\{\mathscr{M}_{\mathscr{W}}\left(r^{\circ}\right), \mathscr{M}_{\mathscr{W}}(\tau)\right\} \\
& \geq \alpha \\
\mathscr{B}_{\mathscr{W}}^{-}\left(u_{1}\right) & \leq \max \left\{\inf \left\{\mathscr{B}_{\mathscr{W}}^{-}\left(t_{1}\right) \mid t_{1} \in u_{1} \diamond v_{1}\right\}, \mathscr{B}_{\mathscr{W}}^{-}\left(\left(v_{1}\right)\right\}\right. \\
& \leq \max \left\{\mathscr{B}_{\mathscr{W}}^{-}\left(t^{\circ}\right), \mathscr{B}_{\mathscr{W}}^{-}\left(v_{1}\right)\right\} \\
& \leq \delta^{-}, \\
\mathscr{B}_{\mathscr{W}}^{+}\left(u_{2}\right) & \geq \min \left\{\sup \left\{\mathscr{B}_{\mathscr{W}}^{+}\left(\dot{t}_{1}\right) \mid \dot{t}_{1} \in u_{2} \diamond v_{2}\right\}, \mathscr{B}_{\mathscr{W}}^{+}\left(v_{2}\right)\right\} \\
& \geq \min \left\{\mathscr{B}_{\mathscr{W}}^{+}\left(\dot{t}_{\circ}\right), \mathscr{B}_{\mathscr{W}}^{+}\left(v_{2}\right)\right\} \\
& \geq \delta^{+}, \\
\mathscr{J}_{\mathscr{W}}(a) & \leq \max \left\{\inf \left\{\mathscr{J}_{\mathscr{W}}(s) \mid s \in a \diamond b\right\}, \mathscr{J}_{\mathscr{W}}(b)\right\} \\
& \leq \max \left\{\mathscr{J}_{\mathscr{W}}\left({ }_{s^{\circ}}\right), \mathscr{J}_{\mathscr{W}}(b)\right\} \\
& \leq \gamma .
\end{aligned}
$$

$\quad \varrho \in U\left(\mathscr{M}_{\mathscr{W}}, \alpha\right), \quad u_{1} \in L\left(\mathscr{S}_{\mathscr{W}}, \delta\right) u_{2} \in L$ $\left(\mathscr{B}_{\mathscr{W}}^{+}, \delta^{+}\right)$, and $a \in L\left(\mathscr{J}_{\mathscr{W}}, \gamma\right)$. Therefore, $U\left(\mathscr{M}_{\mathscr{W}}, \alpha\right)$, $L\left(\mathscr{B}_{\mathscr{W}}^{-}, \delta^{-}\right), U\left(\mathscr{B}_{\mathscr{W}}^{+}, \delta^{+}\right)$, and $L\left(\mathscr{J}_{\mathscr{W}}, \gamma\right)$ are $\mathcal{S} \nVdash-B C K$-ideals

Theorem 8. Let $\mathscr{W}=\left(\mathscr{M}_{\mathscr{W}}, \widetilde{\mathscr{B}}_{\mathscr{W}}, \mathscr{J}_{\mathscr{W}}\right)$ be an MBJ-NS over $\widetilde{\mathscr{H}}$ which satisfies the following condition:

For any $\quad \varrho, \tau, u_{1}, v_{1}, u_{2}, v_{2}, w, z \in \tilde{\mathscr{H}}, \quad$ taking $r=\min \left\{\sup \left\{\mathscr{M}_{\mathscr{W}}(a) \mid a \in \varrho \diamond \tau\right\}, \mathscr{M}_{\mathscr{W}}(\tau)\right\}, \quad t=\max \{\inf$ $\left.\left\{\mathscr{B}_{\mathscr{W}}^{-}(b) \mid b \in u_{1} \diamond v_{1}\right\}, \mathscr{B}_{\mathscr{W}}^{-}\left(v_{1}\right)\right\}, t=\min \left\{\sup \left\{\mathscr{B}_{\mathscr{W}}^{+}(c) \mid c \in u_{2}\right.\right.$ $\left.\left.\diamond v_{2}\right\}, \mathscr{B}_{\mathscr{W}}^{+}\left(v_{2}\right)\right\}$, and $s=\max \left\{\inf \left\{\mathscr{J}_{\mathscr{W}}(d) \mid d \in w \diamond z\right\}, \mathscr{J}\right.$ $\mathscr{W}(z)\}$. Then, by assumption, $U\left(\mathscr{M}_{\mathscr{W}}, \alpha\right), L\left(\mathscr{B}_{\mathscr{W}}^{-}, \delta^{-}\right)$, $U\left(\mathscr{B}_{\mathscr{W}}^{+}, \delta^{+}\right)$, and $L\left(\mathscr{J}_{\mathscr{W}}, \gamma\right)$ are strong hyper-BCK-ideals of $\tilde{\mathscr{H}}$. Condition (3) implies that there exist $a^{\circ} \in \varrho \diamond \tau, b^{\circ} \in u_{1} \diamond v_{1}$, $c^{\circ} \in u_{2} \diamond v_{2}$, and $d^{\circ} \in w \diamond z$ such that $\mathscr{M}_{\mathscr{W}}\left(a_{a^{\circ}}\right)=$ $\sup \left\{\mathscr{M}_{\mathscr{W}}(a) \mid a \in \varrho \diamond \tau\right\}, \mathscr{B}_{\mathscr{W}}^{-}\left(b^{\circ}\right)=\inf \left\{\mathscr{B}_{\mathscr{W}}^{-}(b) \mid b \in u_{1} \diamond v_{1}\right\}$, $\mathscr{B}_{\mathscr{W}}^{+}\left(c^{\circ}\right)=\sup \left\{\mathscr{B}_{\mathscr{W}}^{+}(c) \mid c \in u_{2} \diamond v_{2}\right\}, \quad$ and $\mathscr{J}_{\mathscr{W}}\left(d^{\circ}\right)=$ $\sup \left\{\mathscr{J}_{\mathscr{W}}(d) \mid d \in w \diamond z\right\}$. Hence, 


$$
\begin{aligned}
& \mathscr{M}_{\mathscr{W}}\left(a^{\circ}\right)=\sup \left\{\mathscr{M}_{\mathscr{W}}(a) \mid a \in \varrho \diamond \tau\right\} \geq \min \left\{\sup \left\{\mathscr{M}_{\mathscr{W}}(a) \mid a \in \varrho \diamond \tau\right\}, \mathscr{M}_{\mathscr{W}}(\tau)\right\}=r, \\
& \mathscr{B}_{\mathscr{W}}^{-}\left(b^{\circ}\right)=\inf \left\{\mathscr{B}_{\mathscr{W}}^{-}(b) \mid b \in u_{1} \diamond v_{1}\right\} \leq \max \left\{\inf \left\{\mathscr{B}_{\mathscr{W}}^{-}(b) \mid b \in u_{1} \diamond v_{1}\right\}, \mathscr{B}_{\mathscr{W}}^{-}\left(v_{1}\right)\right\}=t, \\
& \mathscr{B}_{\mathscr{W}}^{+}\left(c^{\circ}\right)=\sup \left\{\mathscr{B}_{\mathscr{W}}^{+}(c) \mid c \in u_{2} \diamond v_{2}\right\} \geq \min \left\{\sup \left\{\mathscr{B}_{\mathscr{W}}^{+}(c) \mid c \in u_{2} \diamond v_{2}\right\}, \mathscr{B}_{\mathscr{W}}^{+}\left(v_{2}\right)\right\}=t, \\
& \mathscr{J}_{\mathscr{W}}\left(d^{\circ}\right)=\inf \left\{\mathscr{J}_{\mathscr{W}}(d) \mid d \in w \diamond z\right\} \leq \max \left\{\inf \left\{\mathscr{J}_{\mathscr{W}}(d) \mid d \in w \diamond z\right\}, \mathscr{M}_{\mathscr{W}}(z)\right\}=s .
\end{aligned}
$$

This imply that $a^{0} \in U\left(\mathscr{M}_{\mathscr{W}}, \alpha\right), \quad b^{\circ} \in L\left(\mathscr{B}_{\mathscr{W}}^{-}, \delta^{-}\right)$, $c^{\circ} \in U\left(\mathscr{B}_{\mathscr{W}}^{+}, \delta^{+}\right)$, and $d^{\circ} \in L\left(\mathscr{J}_{\mathscr{W}}, \gamma\right)$. Hence, $(\varrho \diamond \tau) \cap U\left(\mathscr{M}_{\mathscr{W}}, r\right) \neq \phi, \quad\left(u_{1} \diamond v_{1}\right) \cap L\left(\mathscr{B}_{\mathscr{W}}^{-}, t\right) \neq \phi$, $\left(u_{2} \diamond v_{2}\right) \cap U\left(\mathscr{B}_{\mathscr{W}}^{+}, t\right) \neq \phi,(w \diamond z) \cap L\left(\mathscr{F}_{\mathscr{W}}, s\right) \neq \phi$, and thus $\varrho \in U\left(\mathscr{M}_{\mathscr{W}}, r\right), \quad u_{1} \in L\left(\mathscr{B}_{\mathscr{W}}^{-}, t\right) u_{2} \in U\left(\mathscr{B}_{\mathscr{W}}^{+}, t\right), \quad$ and $w \in L\left(\mathscr{F}_{\mathscr{W}}, s\right)$. It follows that

$$
\begin{aligned}
\mathscr{M}_{\mathscr{W}}(\varrho) \geq r & =\min \left\{\sup \left\{\mathscr{M}_{\mathscr{W}}(a) \mid a \in \varrho \diamond \tau\right\}, \mathscr{M}_{\mathscr{W}}(\tau)\right\}, \\
\mathscr{B}_{\mathscr{W}}^{-}\left(u_{1}\right) \leq t & =\max \left\{\inf \left\{\mathscr{B}_{\mathscr{W}}^{-}(b) \mid b \in u_{1} \diamond v_{1}\right\}, \mathscr{B}_{\mathscr{W}}^{-}\left(\left(v_{1}\right)\right\},\right. \\
\mathscr{B}_{\mathscr{W}}^{+}\left(u_{2}\right) \geq t & =\min \left\{\sup \left\{\mathscr{B}_{\mathscr{W}}^{+}(c) \mid c \in u_{2} \diamond v_{2}\right\}, \mathscr{B}_{\mathscr{W}}^{+}\left(v_{2}\right)\right\}, \\
\mathscr{J}_{\mathscr{W}}(d) \leq s & =\max \left\{\inf \left\{\mathscr{F}_{\mathscr{W}}((d) \mid d \in w \diamond z\}, \mathscr{J}_{\mathscr{W}}(z)\right\} .\right.
\end{aligned}
$$

Therefore, $\mathscr{\mathscr { W }}=\left(\mathscr{M}_{\mathscr{W}}, \widetilde{\mathscr{B}}_{\mathscr{W}}, \mathscr{J}_{\mathscr{W}}\right)$ is a BMBJ-N $\mathcal{S} \llbracket$-BCK-ideal of $\tilde{\mathscr{H}}$.

\section{Conclusions}

This paper found a new link between hyperalgebraic structures and $\mathrm{MBJ}-\mathrm{NS}$ s by introducing BMBJ-N $\mathbb{W} \mathbb{B}-\mathrm{BCK}$ ideal, BMBJ-N $s$-WWH-BCK-ideal, and BMBJ-N $\delta \mathbb{H}-B C K-$

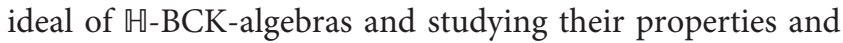
relations. BMBJ-N WVM-BCK-ideals and BMBJ-N $\mathcal{S} \llbracket-B C K-$ ideals in relation to level subsets have been discussed. Conditions for a BMBJ-N WW-D-BCK-ideal to be a BMBJ-N $s$-WW-BCK-ideal have been found. Conditions for an MBJNS to be a BMBJ-N $\mathcal{S}$ \#-BCK-ideal have been given. The results in this paper can be considered as a generalization of the results known for (intuitionistic) fuzzy hyperideals of $\mathbb{M - B C K}$-algebras. In future work, various types of BMBJ-N $\mathbb{M - B C K}$-ideals will be defined and discussed.

\section{Data Availability}

No data were used to support this study.

\section{Conflicts of Interest}

The authors declare that there are no conflicts of interest.

\section{References}

[1] F. Marty, Sur une generalization de la notion de groupe, pp. 45-49, Huitieme congress de Mathematiciens, Scandenaves, Stockholm, 1934

[2] P. Corsini, Prolegomena of Hypergroup Theory, Aviani Editore, Tricesimo, Italy, 1993.

[3] T. Vougiouklis, Hyperstructures and Their Representations, Hadronic Press, Inc., Palm Harbor, FL, USA, 1994.

[4] P. Corsini and V. Leoreanu, Applications of Hyperstructure Theory, Kluwer, Dordrecht, The Netherlands, 2003.
[5] B. Davvaz, Semihypergroup Theory, Academic Press, Cambridge, MA, USA, 1 edition, 2016.

[6] B. Davvaz, Polygroup Theory and Related Systems, World Scientific Publishing Co. Pte. Ltd., Hackensack, NJ, USA, 2013.

[7] B. Davvaz and I. Cristea, Fuzzy Algebraic Hyperstructures An Introduction. Studies in Fuzziness and Soft Computing, Springer, International Publishing, Cham, Switzerland, 2015.

[8] D. Schweigert, "Congruence relations of multialgebras," Discrete Mathematics, vol. 53, pp. 249-253, 1985.

[9] Y. B. Jun, M. M. Zahedi, X. L. Xin, and R. A. Borzoei, "On hyper BCK-algebras," Italian Journal of Pure and Applied Mathematics, vol. 8, pp. 127-136, 2000.

[10] L. A. Zadeh, "Fuzzy sets," Information and Control, vol. 8, no. 3, pp. 338-353, 1965.

[11] K. T. Atanassov, "Intuitionistic fuzzy sets," Fuzzy Sets and Systems, vol. 20, no. 1, pp. 87-96, 1986.

[12] F. Smarandache, Unifying Field in Logics: Neutrosophic Logic. Neutrosophy, Neutrosophic Set, Nutrosophic Probability, American Reserch Press, Rehoboth, NM, USA, 1998.

[13] F. Smarandache, "Neutrosophic set-a generalization of the intuitionistic fuzzy set," International Journal of Pure and Applied Mathematics, vol. 24, pp. 287-297, 2005.

[14] M. M. Takallo, R. A. Borzooei, and Y. B. Jun, "MBJ-neutrosophic structures and its applications in BCK/BCI-algebras," Neutrosophic Sets System, vol. 23, pp. 72-84, 2018.

[15] Y. Jun, S. Kim, and F. Smarandache, "Interval neutrosophic sets with applications in BCK/BCI-algebra," Axioms, vol. 7, no. 2, p. 23, 2018.

[16] A. M. Al-Masarwah and A. G. Ahmad, "On some properties of doubt bipolar fuzzy $\mathrm{H}$-ideals in BCK/BCI-algebras," European Journal of Pure and Applied Mathematics, vol. 11, no. 3, pp. 652-670, 2018.

[17] A. Al-Masarwah and A. G. Ahmad, "Novel concepts of doubt bipolar fuzzy H-ideals of BCK/BCI-algebras," International Journal of Innovative Computing, vol. 14, pp. 2025-2041, 2018.

[18] A. Al-Masarwah and A. G. Ahmad, "m-Polar fuzzy ideals of BCK/BCI-algebras," Journal of King Saud University - Science, vol. 31, no. 4, pp. 1220-1226, 2019.

[19] A. Al-Masarwah and A. G. Ahmad, " $m$-Polar $(\alpha, \beta)$-Fuzzy Ideals in BCK/BCI-Algebras," Symmetry, vol. 11, no. 1, p. 44, 2019.

[20] A. Al-Masarwah, A. G. Ahmad, G. Muhiuddin, and D. AlKadi, "Generalized m-polar fuzzy positive implicative ideals of BCK-algebras," Journal of Mathematics, vol. 2021, Article ID 6610009, 10 pages, 2021.

[21] M. Khan, M. Gulistan, N. Shabir, and S. Muhammad, "Neutrosophic cubic $(\alpha, \beta)$-ideals in semigroups with application $(\alpha, \beta)$-ideals in semigroups with application," Journal of Intelligent \& Fuzzy Systems, vol. 35, no. 2, pp. 2469-2483, 2018.

[22] M. Abu Qamar and N. Hassan, "An approach toward a Q-neutrosophic soft set and its application in decision making," Symmetry, vol. 11, no. 2, p. 139, 2019. 
[23] G. Muhammad, B. Ismat, and Y. Naveed, "A new approach in decision making problems under the environment of neutrosophic cubic soft matrices," Journal of Intelligent \& Fuzzy Systems, vol. 36, no. 1, pp. 295-307, 2019.

[24] M. Khan, M. Gulistan, N. Yaqoob, M. Khan, and F. Smarandache, "Neutrosophic cubic Einstein geometric aggregation operators with application to multi-criteria decision making method," Symmetry, vol. 11, no. 2, p. 247, 2019.

[25] Y. B. Jun and E. H. Roh, "MBJ-neutrosophic ideals of BCK/ BCI-algebras," Open Mathematics, vol. 17, no. 1, pp. 588-601, 2019.

[26] S. Manokaran and M. Prakasam, "On MBJ-Neutrosophic B-Subalgebra,” Neutrosophic Sets System, vol. 28, pp. 216-227, 2019.

[27] M. Khalid, N. A. Khalid, and R. Iqbal, "MBJ-neutrosophic T-ideal on B-algebra," International Journal of Neutrosophic Science, vol. 1, pp. 29-39, 2020.

[28] B. Bordbar, M. M. Takallo, R. A. Borzooei, and Y. B. Jun, "BMBJ-neutrosophic subalgebras in BCI/BCK-algebras," Neutrosophic Sets System, vol. 13, pp. 1-13, 2020.

[29] M. M. Takallo, H. Bordbar, R. A. Borzooei, and Y. B. Jun, "BMBJ-neutrosophic ideals in BCK/BCI-algebras," Neutrosophic Sets System, vol. 27, pp. 1-16, 2019.

[30] R. A. Borzooei, M. M. Takallo, F. Smarandache, and Y. B. Jun, "Positive implicative BMBJ-neutrosophic ideals in BCK-algebras," Neutrosophic Sets System, vol. 23, pp. 126-141, 2018.

[31] Y. B. Jun and X. L. Xin, "Fuzzy hyper BCK-ideals of hyper BCK-algebras," Scientiae Mathematicae Japonicae, vol. 53, pp. 353-360, 2001.

[32] M. Bakhshi, M. M. Zahedi, and R. A. Borzooei, "Fuzzy (positive, weak) implicative hyper BCK-ideals. Iran," International Journal of Fuzzy Systems, vol. 1, no. 2, pp. 63-79, 2004.

[33] R. A. Borzooei and Y. B. Jun, "Intuitionistic fuzzy hyper BCKideals of hyper BCK-ideals. Iran," International Journal of Fuzzy Systems, vol. 1, no. 1, pp. 65-78, 2004.

[34] Y. B. Jun, "Multipolar fuzzy hyper BCK-ideals of hyper BCKalgebras," Journal of Algebraic Hyperstructures and Logical Algebras, vol. 1, no. 1, pp. 37-47, 2020.

[35] Y. J. Seo, H. S. Kim, Y. B. Jun, and S. S. Ahn, "Multipolar intuitionistic fuzzy hyper BCK-ideals in hyper BCK-algebras," Mathematics, vol. 8, no. 8, p. 1373, 2020.

[36] S. Khademan, M. M. Zahedi, R. A. Borzooei, and Y. B. Jun, "Neutrosophic hyper BCK-ideals," Neutrosophic Sets System, vol. 27, pp. 201-217, 2019.

[37] D. Mandal, "Neutrosophic hyperideals of $\Gamma$-semihyperrings," Bulletin of International Mathematical Virtual Institute, vol. 7, pp. 153-164, 2017.

[38] D. Preethi, S. Rajareega, J. Vimala, G. Selvachandran, and F. Florentin Smarandache, "Single-valued neutrosophic hyperrings and hyperideals," Neutrosophic Sets System, vol. 29, pp. 121-128, 2019.

[39] A. A. A. Agboola and B. Davvaz, "Introduction to neutrosophic hypergroups," ROMAI Journal, vol. 9, pp. 1-10, 2013.

[40] Y. B. Jun, X. L. Xin, M. M. Zahedi, and E. H. Roh, "Strong hyper BCK-ideals of hyper BCK-algebras," Japanese Journal of Mathematics, vol. 51, pp. 493-498, 2000.

[41] Y. B. Jun and X. L. Xin, "Scalar elements and hyper atoms of hyper BCK-algebras," Science China Mathematics, vol. 2, pp. 303-309, 1999. 